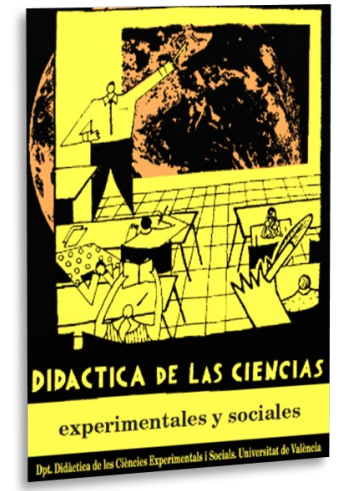

\title{
Evaluación de la adquisición de la competencia científica entre el alumnado de Biología de la ESO y una propuesta para mejorar su habilidad en las representaciones gráficas
}

\author{
Evaluation of the acquisition of scientific \\ competence among Biology students of Compulsory \\ Secondary Education (ESO) and a proposal to \\ improve their skill in graphic representations
}

DOI: $10.7203 / D C E S .41 .19095$

\author{
Antonio Franco López \\ Universidad de Murcia, antoniofrancolopez.af@gmail.com \\ ORCID iD: https://orcid.org/0000-0001-5526-3172 \\ Gabriel Enrique Ayuso Fernández \\ Universidad de Murcia, ayuso@um.es \\ ORCID iD: https://orcid.org/0000-0002-8510-556X \\ Luisa López-Banet \\ Universidad de Murcia, llopezbanet@um.es \\ ORCID iD: https://orcid.org/0000-0002-1951-4242
}

\begin{abstract}
RESUMEN: El desarrollo de la competencia científica en una enseñanza-aprendizaje por indagación basada en problemas cotidianos constituye un planteamiento beneficioso para el aprendizaje de las ciencias. En este trabajo, se analiza la adquisición de diferentes destrezas imprescindibles para la alfabetización científica de estudiantes de $3^{\circ}$ de ESO. Para ello, se ha elaborado un cuestionario que plantea la autopercepción y conocimiento del alumnado de diferentes dimensiones de la competencia científica como identificación de problemas investigables, formulación de hipótesis, diseño experimental, construcción e interpretación de gráficos y elaboración de conclusiones. Los resultados muestran que el alumnado, a pesar de haber realizado actividades relacionadas con estas destrezas y habilidades, presenta serias dificultades para realizar tareas básicas como identificación de problemas, formulación de hipótesis, construcción de gráficas o elaboración de conclusiones. En consecuencia, describimos una propuesta de innovación coherente con la fundamentación teórica y sus resultados, centrada en la mejora de algunos de estos aspectos.
\end{abstract}

Palabras ClaVe: competencia científica, alfabetización científica, nivel competencial, educación secundaria

\begin{abstract}
The development of scientific competence in inquiry-based teaching-learning involving everyday problems constitutes a beneficial approach to science learning. In this work, the acquisition of different essential skills for the scientific literacy of 3rd year students of Compulsory Secondary Education (ESO, for its acronym in Spanish) is analysed. For this purpose, a questionnaire has been developed to assess students' self-perception and knowledge of different dimensions of scientific competence, such as identification of researchable problems, formulation of hypotheses, experimental design, construction and interpretation of graphs, and drawing of conclusions. The results show that the students, despite undertaken out activities related to these skills and abilities, present serious difficulties in carrying out basic tasks such as problem identification, hypotheses formulation, graph construction or drawing conclusions. Consequently, we describe an innovative proposal coherent with the theoretical foundation and its results, focused on improving some of these aspects.
\end{abstract}

KEYWORDS: scientific competence, scientific literacy, competence level, secondary education

Fecha de recepción: marzo de 2021

Fecha de aceptación: noviembre de 2021

Los autores agradecen el proyecto PGC2018-097988-A-I00 financiado por FEDER/Ministerio de Ciencia e Innovación (MCI) de España-Agencia Estatal de Investigación (AEI). 


\section{INTRODUCCIÓN}

La alfabetización científica básica es uno de los objetivos prioritarios que los alumnos y alumnas han de adquirir y desarrollar durante su educación para entender las Ciencias Naturales y poder usarlas como ciudadanos perceptivos, críticos y sensatos (García-Carmona y Acevedo-Díaz, 2018; OCDE, 2006). Esta alfabetización se basa en permitir al alumnado reflexionar, razonar y establecer conexiones que les facilite dar respuesta a todas aquellas situaciones a las que puedan enfrentarse en su vida diaria (Mkimbili y Ødegaard, 2019).

Una de las estrategias más efectivas para promover su aprendizaje se basa en ir desde objetivos generales a acciones específicas y pasar de temas globales a contextos locales a través de problemas socio-científicos (Levrini, Branchetti y Barelli, 2019). Esta perspectiva requiere construir fuertes vínculos entre los contenidos impartidos en clase y los problemas de la comunidad, entre la ciencia escolar y sus experiencias diarias, para que puedan conocer de primera mano cómo ésta influye en aspectos sociales y económicos de su día a día (Campbell, Lubben, y Dlamini., 2000).

Así, el alumnado puede comprender la causa y el efecto de los fenómenos que le rodean, formular preguntas sobre problemas complejos que comprometen a su futuro, elaborar predicciones y desarrollar su parte lógica desde un punto de vista científico. De este modo, es posible poner en marcha un aprendizaje funcional y promover el desarrollo de la competencia científica (GarcíaCarmona, 2008; Levrini et al., 2019).

En este sentido, debemos recordar que la OCDE propuso el término competencia científica, incluyendo en él la capacidad de indagación vinculada a contextos concretos y a la integración de saberes; refiriéndose, entre otros aspectos, al uso que los individuos hacen de los conocimientos científicos para identificar problemas, adquirir nuevos conocimientos, explicar fenómenos científicos y extraer conclusiones basadas en pruebas sobre cuestiones relacionadas con la ciencia (OCDE, 2006). Por ello, algunas investigaciones han profundizado en la delimitación de las dimensiones a considerar dentro de la competencia científica de la investigación escolar, la cual debe relacionarse con las capacidades que el estudiante irá adquiriendo durante su investigación, al justificar el problema, formular objetivos e hipótesis, elaborar un marco teórico y revisar los antecedentes, elaborar un diseño metodológico, presentar y tratar los datos, discutir o valorar los resultados, o emitir conclusiones (Franco-Mariscal, 2015).

En España, las últimas legislaciones educativas incorporan las competencias básicas entre los componentes del currículo; en este aspecto, la anterior ley educativa LOMCE, unificó la matemática y la de conocimiento e interacción con el mundo físico como "Competencia Matemática y Competencias Básicas en Ciencia y Tecnología" (Orden ECD/65, 2015), por lo que, dentro de este marco, tendrían cabida, entre las competencias científicas, el uso e interpretación de las representaciones gráficas por su gran utilidad en la comunicación de la relación entre las variables investigadas (Artola, Mayoral y Benarroch, 2016).

Por otra parte, muchos autores sugieren el uso de metodologías activas sobre retos o problemas cotidianos para el desarrollo de competencias, siendo estos desafíos el pilar principal sobre el que sustentar la enseñanza y el aprendizaje de las ciencias (Franco-Mariscal, Blanco-López y EspañaRamos, 2017; García-Carmona y Acevedo-Díaz, 2018), pues sus resultados son muy satisfactorios a nivel conceptual, procedimental y actitudinal (García-Carmona, 2008).

Desgraciadamente, la situación en las aulas es otra, y esta perspectiva de trabajo difiere significativamente de la que siguen la mayoría de los docentes en sus clases (García-Carmona y Acevedo-Díaz, 2018). Como consecuencia, las capacidades de indagación e investigación del estudiantado no se desarrollan como deberían, como indican numerosos estudios (García-Carmona, 2008; Zúñiga, Leiton y Naranjo, 2014) o los informes PISA (OCDE, 2006).

Entre las dificultades que encuentran los docentes para implantar el modelo planteado destacan la escasez de tiempo, la falta de experiencia para que los proyectos multidisciplinares propuestos resulten exitosos, la priorización de los contenidos meramente declarativos y memorísticos frente a 
los proyectos de investigación y un desconocimiento general sobre cómo orientar las sesiones usando nuevas propuestas didácticas (Franco-Mariscal et al., 2017; García-Carmona y Acevedo-Díaz, 2018).

En este aspecto, debemos considerar que las propuestas de enseñanza de las ciencias basadas en la indagación no deben entenderse "solo" como un fin en sí mismas, es decir, por su utilidad para la adquisición por los estudiantes de destrezas investigativas; sino también, y al mismo tiempo, como un medio para la comprensión del contenido de ciencias (Abd-El-Khalick et al., 2004). De hecho, numerosas propuestas basadas en una metodología de indagación a través de situaciones problemáticas tienen como objetivo principal la consecución del cambio conceptual (por ejemplo, en contenidos de Biología, Ibáñez y Martínez-Aznar, 2005).

A las dificultades del profesorado para seguir una enseñanza basada en la indagación y el planteamiento de problemas debemos sumar las del propio estudiantado: la escasez de compromiso para realizar las actividades grupales, la falta de implicación y participación y su actitud ante las ciencias son algunas de las causas que dificultan su puesta en marcha (Mkimbili y Ødegaard, 2019). $\mathrm{Su}$ falta de destrezas para iniciarse en investigaciones autónomas es otro rasgo remarcable como posible obstáculo. No obstante, ciertos estudios demuestran cómo el uso de entornos adecuados puede ayudar a su desarrollo de forma paulatina (Mkimbili y Ødegaard, 2019).

Por otra parte, las destrezas científicas que promueven los libros de texto de Ciencias Naturales, nos indican la necesidad de mejorar los materiales didácticos habituales y plantear actividades desde un contexto de investigación escolar donde se potencie la autonomía y la indagación de forma gradual. Por el contrario, en los manuales habituales se da poca importancia a la enseñanza de habilidades y destrezas científicas, predominando el exceso de terminología específica y los contenidos más memorísticos muy relacionados con ejercicios que tienen como objetivo la reproducción literal de los mismos. Además, las actividades que siguen el formato de los denominados trabajos prácticos no están diseñadas ni secuenciadas para que los estudiantes puedan aprender los contenidos procedimentales en ellas implicadas (Núñez, Banet y Cordón, 2009).

De acuerdo con estos resultados, muy pocos estudiantes razonan los datos aportados por una investigación usando argumentos lógicos y fundamentados, ya que la mayoría se basan en simples evidencias y observaciones (Ratcliffe, 1999). Por tanto, estos alumnos y alumnas podrían alejarse de la capacidad necesaria para reflexionar y dar respuesta a todos aquellos aspectos sociales y personales influidos por la ciencia que forman parte de sus vidas, como son los problemas medio ambientales, la química verde o las enfermedades que azotan el mundo (García-Carmona, 2008; Mkimbili y Ødegaard, 2019).

Dada su importancia social, personal y curricular, resulta conveniente conocer y analizar el nivel competencial de los y las estudiantes para dilucidar posibles dificultades sobre las que centrar nuestra atención. En consecuencia, este trabajo se presenta como un estudio que pretende investigar y determinar si el alumnado de Educación Secundaria Obligatoria está adquiriendo la competencia científica y las capacidades relacionadas con ésta de forma satisfactoria. Con este fin, se evaluará la adquisición de distintas dimensiones relacionadas con la misma. Se trata, por tanto, de una investigación descriptiva que trata de analizar los niveles de logro alcanzados por estudiantes de Secundaria en diversas dimensiones de la competencia científica y cuyos resultados permitan diseñar una actividad de innovación para mejorar aquellos aspectos que estén menos desarrollados en nuestro alumnado. Para ello, se propondrá la realización de una investigación escolar basada en un problema que sirva de nexo entre la ciencia y la sociedad, buscando incrementar el interés y conectar los contenidos conceptuales, procedimentales y actitudinales.

En suma, nos marcamos como prioridad en este trabajo, una vez se identifiquen en nuestra muestra de estudio las dificultades en las distintas dimensiones de la competencia científica (o subcompetencias), elaborar una propuesta didáctica para mejorar los aprendizajes en los aspectos relacionados con la construcción e interpretación de representaciones gráficas utilizadas en la comunicación de los resultados de una investigación. 


\section{Problemas de la inVestigación Y OBJetivos de la PROPUESTA}

La investigación que mostramos en este trabajo se centra en responder los siguientes interrogantes:

- ¿Qué autopercepción tiene el alumnado de $3^{\circ}$ de la ESO sobre sus destrezas científicas básicas implicadas en una investigación?

- ¿Cuál es el nivel competencial sobre diferentes dimensiones de la competencia científica alcanzado por el alumnado ante determinadas situaciones planteadas?

De acuerdo con el marco teórico que hemos referido, aunque la mayoría del alumnado puede conocer términos y expresiones relacionadas con el mundo de la investigación, como hipótesis o diseño experimental, es probable que los confundan entre sí o no comprendan de forma plena su significado (Cordón, Banet y Nuñez, 2009). De este modo, esperamos que una buena parte de nuestro alumnado de Secundaria no posea las habilidades necesarias para la puesta en práctica de las competencias relacionadas con el trabajo científico, en el contexto de tareas que se puedan plantear en la enseñanza de las ciencias (Cordón, 2008).

En consecuencia, nos planteamos como objetivo del trabajo planificar y diseñar una propuesta de aula para el desarrollo de aquellas dimensiones de la competencia científica (o subcompetencias científicas) que necesiten un mayor refuerzo de acuerdo con nuestra investigación. Se pretende que la propuesta se base en un problema cercano, contextualizado y planificado como una investigación escolar, que pueda ser el motor que genere curiosidad sobre el proceso de enseñanza-aprendizaje y favorezca la adquisición de estas subcompetencias.

\section{Metodología}

En este apartado se describe la muestra de estudio, las características del cuestionario diseñado, los criterios utilizados para el análisis de los resultados obtenidos y las referencias principales para elaborar una propuesta alternativa coherente con nuestro propósito.

\subsection{Descripción de la muestra}

La investigación didáctica presente se llevó a cabo durante el curso 2019-2020 en un centro educativo público de la Región de Murcia ubicado en el centro de la capital, involucrando a 22 alumnos y alumnas de un grupo de $3^{\circ}$ ESO de modalidad ordinario que cursaba la asignatura de "Biología y Geología". El grupo estaba formado por 10 hombres y 12 mujeres con edades entre los 14 y 16 años y un rendimiento académico heterogéneo, según el profesorado del centro.

Se eligió $3^{\circ}$ ESO como curso para realizar esta investigación educativa por ser el último de la Educación Secundaria Obligatoria en el que las asignaturas de ciencias, como Biología y Geología, son de carácter obligatorio. Debido a este motivo, y tras 9 años de escolarización recibidos, el nivel de la competencia básica en matemáticas y básica en ciencia y tecnología debería haber alcanzado un nivel, como mínimo, medio-alto y haber sido asimilada para el final de este curso, puesto que muchos no continuarán sus estudios en este ámbito.

El centro escolar en el que se realiza la investigación está situado en el centro urbano de una capital de tamaño medio-grande. La mayor parte de las familias tienen un nivel socioeconómico medio-alto.

\subsection{Diseño del cuestionario}

Se elaboró un cuestionario que nos permitiera recoger información y analizar las habilidades y destrezas de la muestra de estudiantes para resolver las cuestiones planteadas sobre cada una de las dimensiones de la competencia científica propuestas o subcompetencias. El propósito principal era la 
recopilación de información sobre el nivel competencial en destrezas científicas básicas entre los y las estudiantes: identificación de problemas investigables, formulación de hipótesis, diseño experimental, interpretación y construcción de gráficas y elaboración de conclusiones. Para ello, se usaron como referencia preguntas y planteamientos encontrados en la literatura consultada y validados por los propios autores en sus investigaciones, aunque fueron adaptados para crear un entorno familiar y un contexto conocido por el alumnado (en el Anexo 1, mostramos el cuestionario empleado).

Su estructura constaba de una serie de preguntas de carácter mixto, es decir, preguntas abiertas y tipo test, y en el caso de las últimas, de respuesta única, que fueron agrupadas en 7 apartados (a-g) como puede apreciarse en la Tabla 1.

TABLA 1. Características principales del cuestionario diseñado

\begin{tabular}{cll}
\hline Apartado & \multicolumn{1}{c}{ Subcompetencia evaluada } & \multicolumn{1}{c}{ Fuente } \\
\hline $\mathrm{a}$ & Autopercepciones y cuestiones teóricas & Adaptado de Cordón (2008) \\
\hline $\mathrm{b}$ & Identificación de problemas investigables & $\begin{array}{l}\text { Adaptado de Ferrés-Gurt (2017) y } \\
\text { Cordón (2008) }\end{array}$ \\
\hline $\mathrm{c}$ & Formulación de hipótesis & $\begin{array}{l}\text { Adaptado de Ferrés-Gurt (2017) y } \\
\text { Cordón (2008) }\end{array}$ \\
\hline $\mathrm{d}$ & Diseño experimental & Adaptado de Cordón (2008) \\
\hline $\mathrm{e}$ & Construcción de gráficas & Elaboración propia \\
\hline $\mathrm{f}$ & Interpretación de gráficas & Adaptado de Núñez et al. (2009) \\
\hline $\mathrm{g}$ & Elaboración de conclusiones & Adaptado de Cordón (2008) \\
\hline
\end{tabular}

\subsection{Criterios para el análisis de los resultados del cuestionario}

La evaluación fue realizada por dos investigadores a través de una rúbrica formada por tres categorías con parámetros bien definidos, buscando maximizar la objetividad de los resultados analizados. Estas características aparecen sintetizadas en la Tabla 2.

TABLA 2. Categorías diseñadas para la evaluación del cuestionario

\begin{tabular}{cl}
\hline Categoría & Características de las respuestas \\
\hline $\mathrm{A}$ & $\begin{array}{l}\text { Adecuadas. Aquellas que pueden ser consideradas válidas al ajustarse a lo } \\
\text { pedido en el enunciado de la actividad. }\end{array}$ \\
\hline B & $\begin{array}{l}\text { Inadecuadas. Aquellas que pueden considerarse no adecuadas ya sea por una } \\
\text { confusión entre los elementos del proceso de investigación o por una relación } \\
\text { establecida no coherente con el enunciado. }\end{array}$ \\
\hline $\mathrm{C}$ & Sin contestar \\
\hline
\end{tabular}

\subsection{Diseño de la propuesta educativa}

La propuesta didáctica se dirige al nivel de $3^{\circ}$ de Educación Secundaria Obligatoria (ESO), el mismo que realizó el cuestionario sobre competencia científica. El objetivo de la misma es el de reforzar algunas de las dimensiones de la competencia científica, cuyo nivel de logro entre el alumnado de este curso será estudiado con el cuestionario, y, en especial, los aspectos relacionados con la interpretación y construcción de representaciones gráficas, así como, con la elaboración de conclusiones de una investigación. 


\subsection{Temporalización, secuenciación y consideraciones generales de la propuesta}

Esta propuesta ha sido pensada para realizarse en cuatro clases de 55 minutos (Tabla 3), buscando conectar la ciencia con los problemas sociales y personales de los alumnos y alumnas.

Asimismo, se tendrán en cuenta las características que aparecen explicitadas en nuestro marco teórico para promover la alfabetización científica en el aula, como la resolución de investigaciones escolares a partir de contextos locales (Campbell et al., 2000; Cordón et al., 2009; García-Carmona, 2008; Franco-Mariscal et al., 2017; Levrini et al., 2019).

TABLA 3. Temporalización de referencia de la propuesta de innovación

\begin{tabular}{lcc}
\hline \multicolumn{1}{c}{ Actividad } & Sesión & Temporalización \\
\hline 0. Planteamiento & 1 & $15 \mathrm{~min}$. \\
\hline 1. Bienvenidos al grupo & 1 & $40 \mathrm{~min}$ \\
\hline 2. Los casos se disparan & 2 & $55 \mathrm{~min}$ \\
\hline 3. Dando con el culpable & 3 & $55 \mathrm{~min}$ \\
\hline 4. La edad y el sexo, ¿un factor entre los contagiados? & 4 & $55 \mathrm{~min}$ \\
\hline
\end{tabular}

\section{RESULTADOS E INTERPRETACIÓN}

Los resultados del análisis del formulario han sido divididos en dos secciones bien definidas: autopercepciones sobre la competencia científica y aplicación de las subcompetencias científicas a situaciones concretas. Por otra parte, incluimos en esta sección la descripción de las actividades de la propuesta didáctica elaborada.

\subsection{Autopercepciones sobre la competencia científica}

El bloque I del formulario ("a" en la Tabla 1) tenía como finalidad averiguar si los y las estudiantes recordaban haber realizado actividades donde hubieran tenido que identificar problemas científicos o formular hipótesis. Asimismo, se les preguntó si conocían qué significaban ciertos términos relacionados con los ámbitos de una investigación y las ciencias.

Las primeras 4 preguntas diseñadas fueron de tipo test con cuatro opciones de respuesta posible y una adicional para que el alumnado pudiera incluir su propia respuesta. De esta forma evitamos una posible subjetividad derivada del análisis de los resultados. Además, ello facilita la comparación entre lo que pensaban y sus destrezas científicas.

En el análisis de las respuestas se pudo apreciar cómo la muestra de estudiantes mayoritariamente recordaba haber realizado con mucha o alguna frecuencia actividades relacionadas con la identificación de problemas y la formulación de hipótesis (más de un $70 \%$ en ambos casos) (Tabla 4).

TABLA 4. Autopercepción de la utilización en el aula de diversas subcompetencias científicas por parte de los estudiantes de la investigación $(\mathrm{N}=22)$

\begin{tabular}{cccccc}
\hline Subcompetencia & $\begin{array}{c}\text { Mucha } \\
\text { frecuencia }\end{array}$ & $\begin{array}{c}\text { Poca } \\
\text { frecuencia }\end{array}$ & $\begin{array}{c}\text { No lo } \\
\text { recuerdo }\end{array}$ & $\begin{array}{c}\text { No entiendo } \\
\text { la pregunta }\end{array}$ & $\begin{array}{c}\text { Otra } \\
\text { respuesta }\end{array}$ \\
\hline $\begin{array}{c}\text { Identificación de } \\
\text { problemas }\end{array}$ & 12 & 4 & 6 & 0 & 0 \\
\hline $\begin{array}{c}\text { Formulación de } \\
\text { hipótesis }\end{array}$ & 13 & 5 & 4 & 0 & 0 \\
\hline
\end{tabular}


En cuanto a su grado de conocimiento de los términos "variable" y "diseño experimental", podemos apreciar que más del $75 \%$ de la muestra sabe o cree saber el significado del primer término; si bien, este porcentaje es de un $50 \%$ en lo referido al diseño experimental (Tabla 5).

TABLA 5. Autopercepción del significado de diversas subcompetencias científicas por parte de los estudiantes de la investigación $(\mathrm{N}=22)$

\begin{tabular}{cccccc}
\hline Subcompetencia & $\begin{array}{c}\text { Sí, sé lo que } \\
\text { significa }\end{array}$ & Creo saberlo & $\begin{array}{c}\text { No lo } \\
\text { recuerdo }\end{array}$ & $\begin{array}{c}\text { No sé el } \\
\text { significado }\end{array}$ & $\begin{array}{c}\text { Otra } \\
\text { respuesta }\end{array}$ \\
\hline $\begin{array}{c}\text { Conocimiento de } \\
\text { lo que es una } \\
\text { variable }\end{array}$ & 15 & 2 & 4 & 1 & 0 \\
\hline $\begin{array}{c}\text { Diseño } \\
\text { experimental }\end{array}$ & 9 & 2 & 11 & 0 & 0 \\
\hline
\end{tabular}

Para un análisis algo más preciso del término "diseño experimental" incluimos en el cuestionario una última pregunta en este apartado en la que debían escoger entre cinco situaciones concretas cuál o cuáles consideraban relativas a esta subcompetencia (Tabla 6).

TABLA 6. Significado del término "diseño experimental" para los estudiantes de la investigación (N=22)

\begin{tabular}{lc}
\hline Estamos haciendo un diseño experimental cuando... & $\mathbf{N}^{\mathbf{0}} \mathbf{( \% )}$ \\
\hline $\begin{array}{l}\text { a. ... seguimos el guion que nos ha dado el profesor para observar células } \\
\text { con el microscopio en el laboratorio de Ciencias }\end{array}$ & $3(13,6 \%)$ \\
\hline $\begin{array}{l}\text { b. ... proponemos las actividades a desarrollar para comprobar si es cierta } \\
\text { la hipótesis de que de padres altos nacen hijos altos }\end{array}$ & $9(40,9 \%)$ \\
\hline $\begin{array}{l}\text { c. ... interpretamos los resultados de una experiencia para comprobar el } \\
\text { efecto de la luz sobre el crecimiento de una planta }\end{array}$ & $8(26,2 \%)$ \\
\hline $\begin{array}{l}\text { d. ... clasificamos plantas, con ayuda de unas claves del libro, por la forma } \\
\text { y tamaño de sus hojas }\end{array}$ & $7(31,8 \%)$ \\
\hline No contesta & $2(9,0 \%)$ \\
\hline
\end{tabular}

Como vemos, con respecto al diseño experimental, solo un $40 \%$ del alumnado ( 9 estudiantes) eligió la opción más adecuada, decantándose el resto por otras de las opciones propuestas. Los datos obtenidos coinciden con los obtenidos en la pregunta anterior que nos indican que este término es, en principio, el más difícil de comprender por parte de nuestro alumnado, confundiéndolo con el análisis de los resultados o el seguimiento de un protocolo previamente elaborado. No obstante, el resto de los resultados obtenidos con respecto a su percepción de conocimiento de las subcompetencias de identificación de problemas, formulación de hipótesis o identificación de variables nos podían hacer presuponer un buen dominio de estos aspectos, o al menos, un conocimiento mínimo que les permitiera desenvolverse con cierta soltura en las actividades propuestas en los siguientes apartados del cuestionario.

\subsection{Aplicación de algunas subcompetencias científicas a situaciones concretas}

Las 8 preguntas propuestas en este bloque II del cuestionario (Anexo 1) pretendían que el estudiantado aplicara sus destrezas y conocimientos a problemas científicos reales. En algunas ocasiones, estos planteamientos nos permitieron evaluar varias subcompetencias a la vez, como puede verse en la Tabla 7. 
TABLA 7. Planteamiento de las actividades del cuestionario (Bloque II)

\begin{tabular}{|c|c|c|}
\hline Apartado & Actividades & $\begin{array}{c}\text { Subcompetencias científicas } \\
\text { evaluadas }\end{array}$ \\
\hline $\mathrm{b}$ & $\begin{array}{l}\text { Pregunta } 1 \text {. Estudio de la variación de colesterol } \\
\text { en sangre según la dieta. } \\
\text { Pregunta } 2 \text {. Los factores del medio que influyen } \\
\text { en las cochinillas. } \\
\text { Pregunta } 3 \text {. La experiencia de Redi sobre la } \\
\text { generación espontánea. }\end{array}$ & Identificación de problemas. \\
\hline $\mathrm{c}$ & $\begin{array}{l}\text { Pregunta 2. Los factores del medio que influyen } \\
\text { en las cochinillas. } \\
\text { Pregunta } 3 \text {. La experiencia de Redi sobre la } \\
\text { generación espontánea. } \\
\text { Pregunta } 4 \text {. La relación entre la salinidad y el } \\
\text { punto blanco en peces. }\end{array}$ & Formulación de hipótesis. \\
\hline d & $\begin{array}{l}\text { Pregunta 2. Los factores del medio que influyen } \\
\text { en las cochinillas. } \\
\text { Pregunta 5. Los factores ambientales que } \\
\text { influyen en el caracol. } \\
\text { Pregunta 6. Los factores que influyen en la } \\
\text { germinación. }\end{array}$ & $\begin{array}{l}\text { Propuesta de un diseño } \\
\text { experimental. } \\
\text { Análisis de un diseño } \\
\text { experimental planteado. }\end{array}$ \\
\hline $\mathrm{e}$ & $\begin{array}{l}\text { Pregunta 7. Relación entre altura y edad en } \\
\text { distintos estudiantes. }\end{array}$ & $\begin{array}{l}\text { Elaboración de una gráfica de } \\
\text { dos ejes a partir de una tabla de } \\
\text { dos columnas. }\end{array}$ \\
\hline $\mathrm{f}$ & $\begin{array}{l}\text { Pregunta } 8 \text {. Evolución del consumo de alcohol } \\
\text { entre adolescentes a lo largo del tiempo. }\end{array}$ & $\begin{array}{l}\text { Interpretación de una gráfica } \\
\text { para interpolar datos, indicar el } \\
\text { incremento entre dos puntos y } \\
\text { extrapolar datos. }\end{array}$ \\
\hline $\mathrm{g}$ & $\begin{array}{l}\text { Pregunta 2. Los factores del medio que influyen } \\
\text { en las cochinillas. } \\
\text { Pregunta } 3 \text {. La experiencia de Redi sobre la } \\
\text { generación espontánea. } \\
\text { Pregunta } 8 \text {. Evolución del consumo de alcohol } \\
\text { entre adolescentes a lo largo del tiempo. }\end{array}$ & Elaboración de conclusiones. \\
\hline
\end{tabular}

Los resultados mostrados en la Figura 1, nos han permitido conocer e identificar los aspectos relativos a diferentes habilidades y destrezas relacionadas con la actividad científica con mayores dificultades entre el alumnado de Educación Secundaria Obligatoria.

En la identificación de problemas investigables (Figura 1.1), el principal problema detectado fue la confusión en el alumnado entre las denominadas "preguntas de información", que no llevan a una investigación, y los interrogantes científicos investigables (de un modo similar a lo detectado en el estudio de Ferrés-Gurt, 2017), como puede verse en la Tabla 8 donde se recoge una muestra de las respuestas literales obtenidas. 
FIGURA 1. Resultados del cuestionario aplicando la rúbrica de evaluación con las categorías A (adecuada), B (no adecuada) y C (en blanco)

1. Identificación de problemas investigables

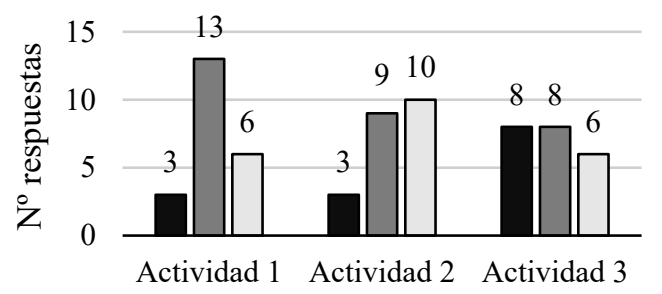

$\square \triangle \mathrm{A} \square \mathrm{C}$

3. Diseño experimental

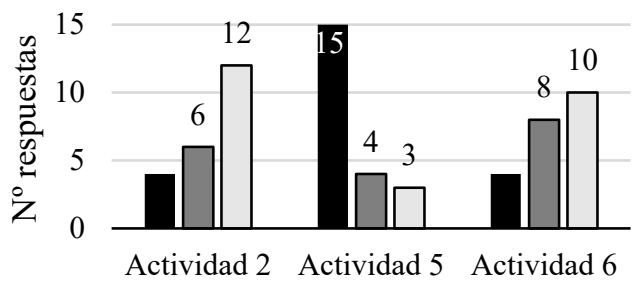

- $\mathrm{A} \square \mathrm{B} \square \mathrm{C}$
2. Formulación de hipótesis

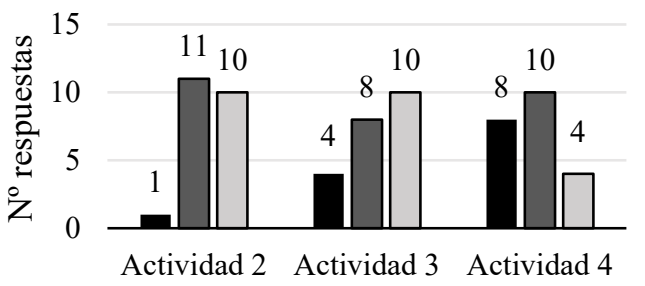

- $\mathrm{A} \square \mathrm{B} \square \mathrm{C}$

\section{Construcción de gráficos simples}

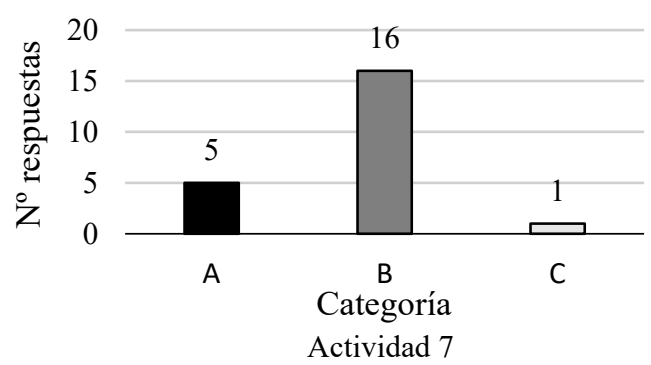

6. Elaboración de conclusiones

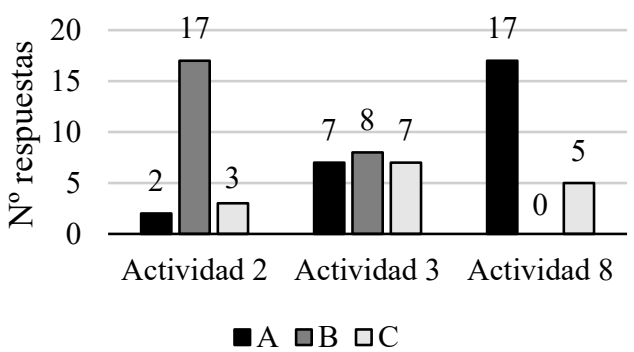

Actividad 8

De forma análoga y de acuerdo con investigaciones similares (Cordón, 2008), en la formulación de hipótesis (Figura 1.2) se apreciaron ciertas dificultades para diferenciar esta subcompetencia de otras, como la identificación de problemas investigables, concentrándose buena parte de las respuestas en la categoría B (inadecuadas). En estos casos, las hipótesis propuestas fueron ambiguas o no se relacionaron realmente con el problema (Tabla 9). De este modo, a pesar de que la mayoría del alumnado decía saber qué era una hipótesis, buena parte del mismo no mostró capacidad suficiente para formularlas.

Con respecto al diseño experimental (Figura 1.3), las respuestas del alumnado a las preguntas 2 y 6 se concentraron en las categorías B y C (81,8\% en ambos casos, 18 de nuestra muestra); si bien, en la cuestión 5 (sobre los factores que pueden influir en el desplazamiento de los caracoles), predominaron las respuestas adecuadas $(68,1 \%, 15$ respuestas) (Tabla 10). Esto no es casual, ya que el grado de complejidad del diseño a proponer en esta actividad era menor al estar condicionado por menos variables que las otras dos situaciones planteadas. 
TABLA 8. Algunas respuestas relacionadas con la identificación de problemas investigables

\begin{tabular}{|c|c|c|}
\hline Preguntas & Ejemplos de respuesta adecuada & $\begin{array}{c}\text { Ejemplos de respuesta no } \\
\text { adecuada }\end{array}$ \\
\hline 1. Dieta & $\begin{array}{l}\text { ¿Qué tipo de grasas aumentan más el } \\
\text { colesterol en sangre, las de origen } \\
\text { animal o aceite de oliva?” (13,6\%) }\end{array}$ & $\begin{array}{l}\text { “Cómo puede aumentar el } \\
\text { colesterol el aceite de oliva y las } \\
\text { grasas animales?” (59,0\%) }\end{array}$ \\
\hline 2. Cochinillas & $\begin{array}{l}\text { “Las cochinillas se esconden por la } \\
\text { luz/oscuridad, por la temperatura, por } \\
\text { ninguna de esas opciones?” (13,6\%) }\end{array}$ & $\begin{array}{l}\text { "Es que cuál es la necesaria para } \\
\text { que una cochinilla viva mejor" } \\
(40,9 \%)\end{array}$ \\
\hline 3. Redi & $\begin{array}{l}\text { "Pretendia resolver si podrian } \\
\text { aparecer insectos procedentes de la } \\
\text { carne sin una hembra que pusiera } \\
\text { huevos." }(36,3 \%)\end{array}$ & $\begin{array}{l}\text { "Cómo podía almacenar comida } \\
\text { sin miedo a ser infectada por las } \\
\text { moscas" }(36,3 \%)\end{array}$ \\
\hline
\end{tabular}

Nota: Entre paréntesis, representatividad, en \%, de la respuesta elegida como ejemplo.

TABLA 9. Algunas respuestas relacionadas con la formulación de hipótesis

\begin{tabular}{|c|c|c|}
\hline Pregunta & Ejemplos de respuesta adecuada & $\begin{array}{c}\text { Ejemplos de respuesta no } \\
\text { adecuada }\end{array}$ \\
\hline 2. Cochinillas & $\begin{array}{l}\text { "Cuanta más oscuro y menos } \\
\text { temperatura haya, saldrá de su } \\
\text { escondite" }(4,5 \%)\end{array}$ & $\begin{array}{l}\text { "Mi hipótesis sería, pondría a las } \\
\text { cochinillas en un recipiente con } \\
\text { mucha luz y con temperaturas bajas, } \\
\text { y después a la semana siguiente las } \\
\text { pondría en la hojarasca a oscuras y } \\
\text { con temperatura alta". (50,0\%) }\end{array}$ \\
\hline 3. Redi & $\begin{array}{l}\text { "Suponemos que las larvas son por } \\
\text { los huevos de las moscas" (18,1\%) }\end{array}$ & $\begin{array}{l}\text { "Si tapamos o cerramos de manera } \\
\text { distinta el envase tendremos distintos } \\
\text { resultados" }(36,3 \%)\end{array}$ \\
\hline 4. Acuario & $\begin{array}{l}\text { "Según mi hipótesis creo que cuánta } \\
\text { más sal pongas durante } 1 \text { minuto, la } \\
\text { enfermedad disminuye hasta el } \\
\text { punto de hacerla desaparecer" } \\
(36,3 \%)\end{array}$ & $\begin{array}{l}\text { "¿Aguantarán los protozoos en agua } \\
\text { salada?" (45,4\%) }\end{array}$ \\
\hline
\end{tabular}

Nota: Entre paréntesis, representatividad, en \%, de la respuesta elegida como ejemplo.

En la construcción de gráficos (Figura 1.4) se observaron errores como la falta de proporcionalidad de los ejes, uno de los ejes invertido, repetición de datos (al copiar los resultados de la tabla tal como aparecen) o dejar la gráfica incompleta o inacabada (Figura 2). Estas situaciones coinciden con otras investigaciones como el estudio de Núñez et al. (2009). Solo 5 estudiantes de nuestra muestra de estudio (en torno al $20 \%$ ) consiguieron dibujar una gráfica de dos ejes de categoría A (adecuada) a partir de una tabla.

En contraste, en la interpretación de gráficas (Figura 1.5), se obtuvieron resultados más satisfactorios en las cuestiones sobre interpolación, incremento entre dos puntos de la gráfica y extrapolación de datos, respectivamente. Por consiguiente, se puede deducir una mayor dificultad entre el alumnado para construir gráficas que para interpretarlas y analizarlas. 
TABLA 10. Algunas respuestas relacionadas con el diseño experimental

\begin{tabular}{|c|c|c|}
\hline Pregunta & Ejemplos de respuesta adecuada & $\begin{array}{c}\text { Ejemplos de respuesta no } \\
\text { adecuada }\end{array}$ \\
\hline 2. Cochinillas & $\begin{array}{l}\text { "Haría } 4 \text { pruebas: } 1 \text {. Hojarasca y un } \\
\text { calentador o algo asi para calentar } \\
\text { (la hojarasca para crear } \\
\text { oscuridad). 2. Hojarasca y las } \\
\text { pondría en un lugar frio. 3. Sin } \\
\text { hojarasca, luz encendida y } \\
\text { calentador. 4. Sin hojarasca y sin } \\
\text { calentador. Después de algún } \\
\text { tiempo, comprobaría en cuál de los } \\
\text { tanques las cochinillas están mejor } \\
\text { y si hay alguna muerta" }(18,1 \%)\end{array}$ & $\begin{array}{l}\text { "Movería las cochinillas primero a } \\
\text { un sitio con luz y luego a otro con } \\
\text { oscuridad para ver cómo actúan. A } \\
\text { continuación, con una lámpara le } \\
\text { daría calor hasta unos } 30^{\circ} \text { y luego } \\
\text { probaría a meterlo en un sitio frío con } \\
10^{\circ} "(27,2 \%)\end{array}$ \\
\hline 5. Caracol & $\begin{array}{l}\text { "Por una parte, sacaría al caracol } \\
\text { de ese círculo y lo metería y } \\
\text { rodearía de agua y luego, en otro } \\
\text { momento por sal, y, comprobaría } \\
\text { cual de esos circulos atraviesa" } \\
(68,1 \%)\end{array}$ & $\begin{array}{l}\text { "Creo que el problema es de la sal ya } \\
\text { que el caracol es muy baboso y para } \\
\text { ser baboso necesita mucha agua } \\
(\ldots) \text { ". (18,1\%) }\end{array}$ \\
\hline 6.Germinación & $\begin{array}{l}\text { "En que deberían dividir todas las } \\
\text { semillas, ponerlas a diferentes } \\
\text { temperaturas para ver cuáles } \\
\text { germinan o cuáles no, según la } \\
\text { temperatura" }(18,1 \%)\end{array}$ & $\begin{array}{l}\text { "En que no germinaron todas las } \\
\text { plantas, con lo cual no se ha cumplido } \\
\text { el experimento" }(36,3 \%)\end{array}$ \\
\hline
\end{tabular}

Nota: Entre paréntesis, representatividad, en \%, de la respuesta elegida como ejemplo.

Por último, se constató una mayor facilidad para elaborar conclusiones a partir de gráficos simples (actividad 8), con cerca de un $75 \%$ de respuestas consideradas adecuadas científicamente, que al establecer conclusiones partiendo de un texto (actividades 2 y 3 ) (Figura 1.6). De este modo, consideramos que se produce, por parte de nuestra muestra de estudiantes, un mejor entendimiento del lenguaje visual y gráfico que del escrito (Tabla 11). Esta cuestión se considera de interés a la hora de la presentación de datos al estudiantado.

FIGURA 2. Algunos ejemplos de representaciones gráficas no adecuadas

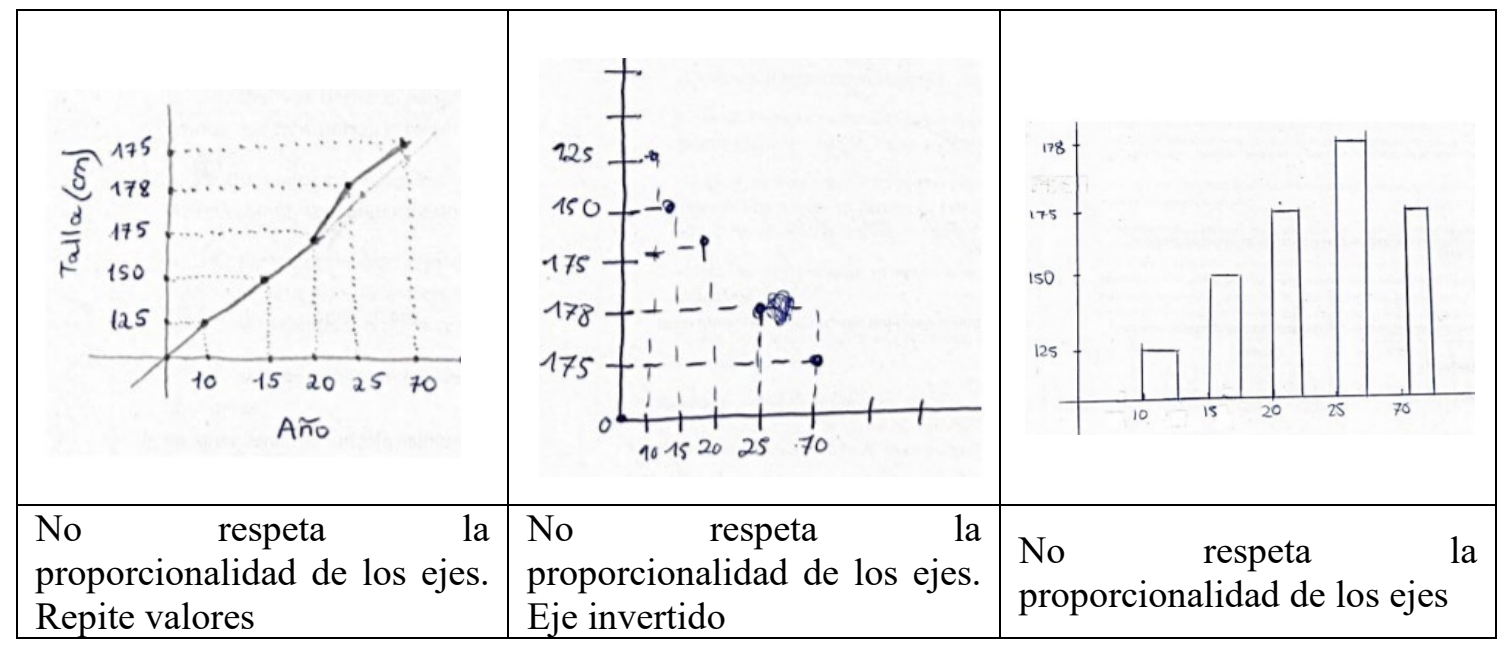


En relación a este último aspecto, al analizar globalmente todo el cuestionario, hemos apreciado entre nuestros estudiantes una cierta dificultad lingüística para expresar sus ideas, por lo que consideramos que esta carencia puede influir en los bajos resultados obtenidos en el conjunto de las subcompetencias estudiadas.

TABLA 11. Algunas respuestas relacionadas con la elaboración de conclusiones

\begin{tabular}{|c|c|c|}
\hline Pregunta & Ejemplos de respuesta adecuada & $\begin{array}{c}\text { Ejemplos de respuesta no } \\
\text { adecuada }\end{array}$ \\
\hline 2. Cochinillas & $\begin{array}{l}\text { “(.) me basaría en cómo } \\
\text { reaccionan a los cambios y a las que } \\
\text { siguen vivas" }(9,0 \%)\end{array}$ & $\begin{array}{l}\text { "Llegaría a que las cochinillas solo } \\
\text { salen de dia y a una temperatura } \\
\text { alta" }(77,2 \%)\end{array}$ \\
\hline 3. Redi & $\begin{array}{l}\text { "Los insectos no aparecen en la } \\
\text { carne putrefacta por arte de magia, } \\
\text { sino que necesitan depositar sus } \\
\text { huevos para que nazcan larvas" } \\
(31,8 \%)\end{array}$ & $\begin{array}{l}\text { "Que aparecieron larvas", "que no } \\
\text { salían larvas" (36,3\%) }\end{array}$ \\
\hline 8. Gráficas & $\begin{array}{l}\text { "Cada vez beben más alcohol los } \\
\text { jóvenes", "ha ido aumentando" } \\
(77,2 \%)\end{array}$ & -- \\
\hline
\end{tabular}

Nota: Entre paréntesis, representatividad, en \%, de la respuesta elegida como ejemplo.

Los resultados que hemos obtenido en nuestra investigación coinciden, como hemos señalado, con los obtenidos por otros trabajos y ponen de manifiesto la dificultad de nuestros estudiantes de Secundaria a la hora de identificar problemas de una investigación, formular hipótesis, diseñar experiencias, realizar gráficas o establecer conclusiones. Estos resultados contrastan más aún con la favorable autopercepción general señalada por los mismos estudiantes con respecto a estas subcompetencias.

\subsection{Descripción de la propuesta y materiales de la innovación}

Como consecuencia de los resultados expuestos y de acuerdo con los objetivos explicitados, hemos diseñado una propuesta de innovación para desarrollar una de las subcompetencias que acumuló un mayor número de respuestas de categoría $\mathrm{B}$ y $\mathrm{C}$ en el formulario: la construcción de gráficas simples. Igualmente, a través de esta propuesta se trabajará la elaboración de conclusiones a partir de datos y representaciones, con el fin de promover una evolución en las conclusiones simplistas o parciales y fomentar las capacidades lingüísticas escritas para seleccionar información científica relevante.

La propuesta que aquí presentamos, la hemos titulado "La epidemia que azota nuestro municipio" y está dirigida al alumnado del mismo nivel educativo en el que hicimos nuestra investigación sobre su conocimiento y utilización de las subcompetencias científicas, $3^{\circ}$ de la ESO.

El escenario elegido como trasfondo versa sobre el SARS-Cov-2 y sus características epidemiológicas, un tema que por su trascendencia social actual puede captar la atención de nuestro alumnado. En esta se propone un ciclo en el que se plantea el problema, una serie de acciones para entender y profundizar en él y un desenlace donde se vuelve al punto inicial para poder dar una respuesta con argumentos sólidos y fundamentados. Además, en la propuesta se establece una gradación en la autonomía del alumnado conforme se va avanzando en ella. En la Tabla 12 mostramos un resumen de la propuesta señalando el título de cada actividad, el desarrollo previsto en el aula y los objetivos relacionados con las diferentes dimensiones de la competencia científica trabajadas. En los Anexos 2-5, reproducimos algunas de las actividades propuestas. 
En el inicio de la misma, se pide al alumnado que trabajando en pequeños grupos (4-5 miembros) lean una carta en la que se les plantea la actividad problemática y se les solicita su ayuda por una posible epidemia en la zona donde viven. Junto a ella, dispondrán de la información básica de los 10 primeros pacientes hospitalizados (sexo, edad y unos pocos síntomas). En esta parte, deberán redactar una respuesta a la carta donde destaquen los aspectos que más les hayan llamado la atención de la situación planteada y una primera hipótesis sobre la causa del problema.

TABLA 12. Esquema de la propuesta didáctica elaborada

\begin{tabular}{|c|c|c|}
\hline Título & Desarrollo de la actividad & $\begin{array}{c}\text { Objetivos y subcompetencias } \\
\text { a trabajar } \\
\end{array}$ \\
\hline $\begin{array}{c}0 . \\
\text { Planteamiento } \\
\text { del problema }\end{array}$ & $\begin{array}{l}\text { Lectura de la carta y tabla de pacientes. } \\
\text { Respuesta a la misiva señalando los rasgos } \\
\text { destacables y formulación de una } \\
\text { hipótesis sobre el problema. }\end{array}$ & 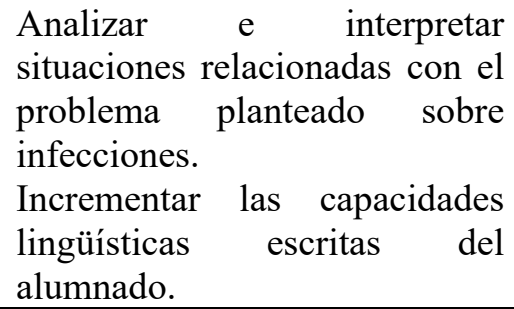 \\
\hline $\begin{array}{r}\text { 1. Bienve } \\
\text { al gru }\end{array}$ & $\begin{array}{l}\text { Respuesta a cuestiones sobre } \\
\text { proporcionalidad y asignación de } \\
\text { variables a los ejes cartesianos usando un } \\
\text { contexto epidemiológico. }\end{array}$ & $\begin{array}{l}\text { Mejorar la habilidad para } \\
\text { construir gráficas y tablas } \\
\text { simples. }\end{array}$ \\
\hline $\begin{array}{l}\text { 2. Los casos se } \\
\text { disparan }\end{array}$ & $\begin{array}{l}\text { Dibujo de gráficas a través de preguntas } \\
\text { guía para favorecer el debate. } \\
\text { Estudio de varias zonas geográficas y } \\
\text { diferentes lugares donde se han detectado } \\
\text { infecciones. } \\
\text { Escrito al vecindario de la zona } \\
\text { explicando la gravedad de la situación. }\end{array}$ & $\begin{array}{l}\text { Mejorar la habilidad para } \\
\text { construir gráficas y tablas } \\
\text { simples. } \\
\text { Analizar e interpretar } \\
\text { situaciones relacionadas con las } \\
\text { infecciones. } \\
\text { Incrementar las capacidades } \\
\text { lingüísticas escritas del } \\
\text { alumnado. }\end{array}$ \\
\hline $\begin{array}{l}\text { 3. Dan } \\
\text { el cul }\end{array}$ & $\begin{array}{l}\text { Señalar el agente patógeno usando la tabla } \\
\text { de pacientes actualizada y cuatro } \\
\text { flashcard con información sobre } \\
\text { patologías con síntomas comunes. } \\
\text { Redacción de un documento que relacione } \\
\text { la información aportada. } \\
\text { Proposición de una conclusión sobre la } \\
\text { actividad. }\end{array}$ & $\begin{array}{l}\text { Analizar e interpretar } \\
\text { situaciones relacionadas con el } \\
\text { problema planteado sobre } \\
\text { infecciones. } \\
\text { Incrementar las capacidades } \\
\text { lingüísticas escritas del } \\
\text { alumnado. } \\
\text { Trabajar la elaboración de } \\
\text { conclusiones a partir de datos y } \\
\text { representaciones }\end{array}$ \\
\hline $\begin{array}{l}\text { 4. La edad y el } \\
\text { sexo, ¿un } \\
\text { factor entre los } \\
\text { contagiados? }\end{array}$ & $\begin{array}{l}\text { Realización de gráficas de forma } \\
\text { autónoma. } \\
\text { Comparación de la información de las } \\
\text { gráficas con la recibida en otros puntos del } \\
\text { problema. } \\
\text { Elaboración de la conclusión final sobre el } \\
\text { problema. }\end{array}$ & 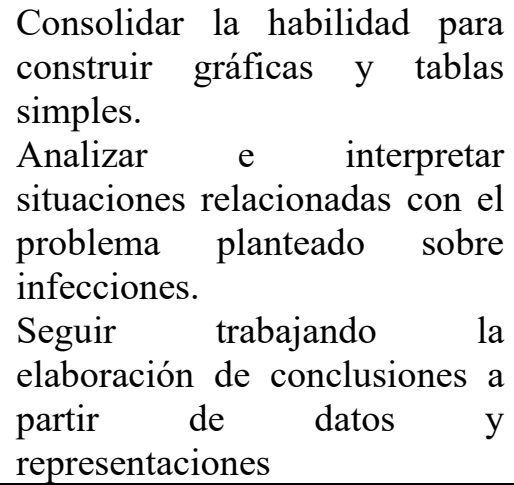 \\
\hline
\end{tabular}


A continuación, se les proporcionará el material de la Actividad 1 "Bienvenidos al grupo" (Anexo 2) donde se trabajará la asignación de variables a los ejes, la proporcionalidad y la escala. La organización de los grupos del planteamiento se mantendrá a lo largo de toda la intervención.

En la primera parte de la Actividad 1, se dispondrá de 5 gráficas (A-E) para que se indique cuál representa el número de personas afectadas según su edad. Para dar con la respuesta correcta y descartar el resto, deberán tener en cuenta las variables que aparecen en los ejes. Además, para profundizar en su decisión y hacer reflexionar al grupo de estudiantes, se plantean cuestiones como “¿en qué os habéis basado para elegir esa opción y descartar las otras?” o “¿qué información obtendrías de cada una de las gráficas anteriores según los ejes?"

En la segunda parte, se dispondrá de 3 gráficas para justificar cuáles representan la misma información. Para ello, es necesario que las gráficas que se les presenten tengan, por ejemplo, distinta escala en el eje de ordenadas. Como es posible que el alumnado no repare en un primer momento en los ejes cartesianos, se plantean cuestiones como "¿qué indica cada gráfica? (apóyate en los datos)" para guiarlos.

En la Actividad 2 "Los casos se disparan" (Anexo 3), se profundizará en la habilidad para dibujar gráficos. Un aumento de casos en zonas cercanas a su lugar de residencia será el epicentro que protagonice la actividad, siendo la tarea principal, la construcción de una gráfica a partir de la información aportada en el enunciado (número de casos por comarca). Primero deberán responder de forma individual y por escrito a unas cuantas cuestiones; y, posteriormente, dibujar una gráfica usando una serie de preguntas guía diseñadas a partir de los errores detectados en nuestro cuestionario.

Después, se les plantean 4 lugares que sufrieron masificaciones unos días antes del aumento de los casos (cine, fiestas privadas, institutos y jardines), para que justifiquen cuál es el foco de infección más importante. Así, deben responder a diferentes cuestiones, para lo que deberán comparar las comarcas más afectadas con los lugares propuestos y el número de personas contagiadas frente al número de personas que acudieron.

Finalmente, para seguir desarrollando su capacidad para expresarse de forma escrita, se deberá escribir una misiva a la ciudadanía explicándoles la situación, las causas y las consecuencias y otros datos que consideren relevantes.

En la Actividad 3 "Dando con el culpable" recibirán la información actualizada de los 10 primeros hospitalizados y 4 posibles agentes causales (Anexo 4). En grupos deberán analizar los signos y síntomas de cada paciente, justificar cuál es el agente causal implicado y el lugar o zona donde pudo haber empezado. También se les pide que preparen un escrito que conecte toda la información del problema y comenten las conclusiones que han podido extraer hasta el momento con la información que se les ha ofrecido.

Por último, en la Actividad 4 "La edad y el sexo, ¿un factor entre los contagiados?” (Anexo 5) deberán construir gráficos a partir de tablas de 2 columnas y compararlos con la información del planteamiento para deducir factores epidemiológicos de interés, como las edades y sexos de las personas afectadas.

Para terminar, se deberá proponer una conclusión sobre el problema; y reflexionar sobre los principales aspectos que han aprendido con la actividad y qué contenidos relacionados podrían seguir estudiando para poder guiar futuros proyectos con los y las estudiantes.

\section{CONCLUSIONES E IMPLICACIONES DIDÁCTICAS}

Como han puesto en evidencia los resultados presentados, la compresión de los elementos relacionados con una investigación científica es insuficiente por parte de los estudiantes de nuestra muestra de estudio. Aunque podrían surgir distintas interpretaciones, todo parece apuntar a que estos contenidos no se trabajan en el aula de la mejor manera posible, impidiendo que se desarrollen como deberían. Además, destaca la falta de actividades en los libros de texto sobre estos aspectos 
relacionados con la competencia científica y una secuenciación adecuada de estos contenidos (Cordón et al., 2009; Vázquez y Manassero, 2012).

Podría resultar llamativo que los alumnos y alumnas declarasen haber realizado frecuentemente tareas relacionadas con la identificación de problemas científicos y la formulación de hipótesis, entre otros, en la primera parte del cuestionario. Sin embargo, es probable que el estudiantado crea estar formulando hipótesis cuando en realidad se dedica a tareas más rutinarias o ejercicios de lápiz y papel de tipo memorístico. Así mismo, hemos podido comprobar en nuestro trabajo que conocer la denominación de un término científico, por parte de los y las estudiantes, no implica tener la destreza a la que se hace referencia.

Encontramos dificultades por nuestros estudiantes en todas las subcompetencias analizadas (identificación de problemas en una investigación, formulación de hipótesis, diseño de experiencias, realización de gráficas o establecimiento de conclusiones), si bien debemos destacar que estas dificultades pueden ser menores en función del contexto problemático planteado, en el que pueden influir aspectos como la familiaridad con la situación o el número de variables implicadas.

En consecuencia, se plantea la necesidad de programar y promover actividades orientadas a suplir dichas carencias y a alcanzar los objetivos marcados para este nivel de enseñanza, prestando especial atención a las dificultades y obstáculos apreciados. No tiene sentido seguir promoviendo una educación basada principalmente en el aprendizaje memorístico donde predomina la enseñanza de términos y conceptos sin conexión. Es necesario promover el aprendizaje de estos conceptos junto con otros contenidos de tipo procedimental y actitudinal, aspectos que pueden ayudar al alumnado a contemplar y entender las ciencias como una herramienta útil y valiosa (Cordón et al., 2009; GarcíaCarmona, 2008; García-Carmona y Acevedo-Díaz, 2018). De este modo, invitar a los y las estudiantes a reflexionar sobre los fenómenos que se trabajan en el aula se hace casi imprescindible. Para ello, la creación de entornos adecuados para su promoción es fundamental pues, como se ha podido observar, llegan a influir en el estudiantado de forma notable impidiéndole o facilitándole el desarrollo y aplicación de sus destrezas.

Además, aunque no era uno de los objetivos principales de esta investigación, también se han podido apreciar carencias a nivel gramatical y de vocabulario a través de las preguntas de respuesta abierta.

Igualmente, aunque abogamos por el uso de problemas o situaciones cotidianas como motor principal, ello no implica que no puedan usarse otros planteamientos para el desarrollo de la competencia científica y que estos sean igualmente satisfactorios. Disponer de materiales didácticos diseñados con esta finalidad y concebidos como una investigación escolar, también podría ser de mucha utilidad para alcanzar esta meta.

En el caso de no adoptar medidas que terminen con la situación expuesta en este trabajo, nuestros estudiantes podrían no tener las habilidades necesarias para reflexionar y apreciar todos aquellos aspectos sociales y personales que están influidos por la ciencia (Zúñiga et al., 2014). Para ello, su implicación en investigaciones holísticas donde puedan explorar y comprender los contenidos conceptuales, al tiempo que se introducen en el desarrollo de habilidades relativas a las distintas dimensiones de la competencia científica, conectando con sus ideas e intereses (Cordón, 2008), podría ser un buen camino a seguir para desarrollar por completo este objetivo.

Por ello, en la propuesta de innovación presentada destacan dos mejoras, a nuestro juicio, de importancia frente a la enseñanza tradicional: el empleo de una problemática cercana como forma de mejorar la motivación e interés por el aprendizaje de las enfermedades infecciosas, la Inmunología y la Microbiología, y la inclusión en las propuestas de enseñanza de ciertas habilidades y destrezas básicas relacionados con el desarrollo de la competencia científica entre el alumnado. 


\section{Perspectivas de LA INVESTIGACIÓN}

Por último, resulta pertinente señalar que además de analizar el éxito o fracaso de las propuestas presentadas, futuras investigaciones educativas podrían ir encaminadas a:

- Continuar esta línea de investigación, ampliando el tamaño de la muestra buscando obtener datos más representativos y así poder compararlos con los obtenidos en este trabajo. En este aspecto, consideramos que debemos profundizar en el análisis de los niveles de logro del alumnado en cada una de las subcompetencias científicas estudiadas, ampliando dichos niveles de acuerdo con las propuestas de otros investigadores (Ferrés-Gurt, Marbá y Sanmartí, 2015). - Analizar el impacto de estos resultados en el aprendizaje del alumnado y en su actitud frente a las ciencias.

- Proponer líneas de desarrollo curricular que, teniendo en cuenta los datos presentados, estudien y analicen la eficacia de los recursos didácticos más utilizados en las aulas para el desarrollo de la competencia científica y así permitan orientar nuevas estrategias educativas.

\section{Referencias}

Abd-El-Khalick, F., Boujaoude, S., Duschl, R., Lederman, N. G., Mamlok-Naaman, R., Hofstein, A., Niaz, M., Treagust, D. y Tuan, H. L. (2004). Inquiry in science education: International perspectives. Science Education, 88(3), 397-419. DOI: 10.1002/sce.10118

Artola, E. C., Mayoral, L. E. y Benarroch, A. (2016). Dificultades de aprendizaje de las representaciones gráficas cartesianas asociadas a biología de poblaciones en estudiantes de educación secundaria. Un estudio semiótico. Revista Eureka sobre Enseñanza y Divulgación de las Ciencias, 13(1), 36-52. DOI: 10498/18012

Campbell, B., Lubben, F. y Dlamini, Z. (2000). Learning science through contexts: Helping pupils make sense of everyday situations. International Journal of Science Education, 22(3), 239-252. DOI: $10.1080 / 095006900289859$

Cordón, R. (2008). Enseñanza y aprendizaje de procedimientos científicos (contenidos procedimentales) en la Educación Secundaria Obligatoria: Análisis de la situación, dificultades y perspectivas (Tesis Doctoral).

Cordón, R., Banet, E. y Nuñez, F. (2009). Las habilidades científicas en los libros de texto. Enseñanza de las Ciencias: Revista de Investigación y Experiencias Didácticas, (Extra), 861-868.

Ferrés-Gurt, C. (2017). El reto de plantear preguntas científicas investigables. Revista Eureka sobre Enseñanza y Divulgación de las Ciencias, 14(2), 410-426. DOI: 10.25267/rev_eureka_ensen_divulg_cienc.2017.v14.i2.09

Ferrés-Gurt, C., Marbá, A. y Sanmartí, N. (2015). Evaluación de la competencia de indagación científica de los bachilleres. Revista Eureka sobre Enseñanza y Divulgación de las Ciencias, 12(1), 22-37. DOI: 10.25267/Rev_Eureka_ensen_divulg_cienc.2015.v12.i1.03

Franco-Mariscal, A. J. (2015) Competencias científicas en la enseñanza y el aprendizaje por investigación. Un estudio de caso sobre corrosión de metales en secundaria. Enseñanza de las Ciencias: Revista de Investigación y Experiencias Didácticas, 33(2), 231-252. DOI: 10.5565/rev/ensciencias. 1645

Franco-Mariscal, A. J., Blanco-López, Á. y España-Ramos, E. (2017). Diseño de actividades para el desarrollo de competencias científicas. Utilización del marco de PISA en un contexto relacionado con la salud. Revista Eureka sobre Enseñanza y Divulgación de las Ciencias, 14(1), 38-53. DOI: 10.25267/rev_eureka_ensen_divulg_cienc.2017.v14.i1.04

García-Carmona, A. (2008). Relaciones CTS en la Educación Científica Básica. II. Investigando los problemas del mundo. Enseñanza de las Ciencias: Revista de Investigación y Experiencias Didácticas, 26(3), 389-402. DOI: 10.5565/rev/ensciencias.3750 
García-Carmona, A. y Acevedo-Díaz, J. A. (2018). The Nature of Scientific Practice and Science Education: Rationale of a Set of Essential Pedagogical Principles. Science and Education, 27(5), 435-455. DOI: 10.1007/s11191-018-9984-9

Ibáñez, T. y Martínez-Aznar, M. (2005). Solving Problems in Genetics II: Conceptual restructuring, International Journal of Science Education, 27(12), 1495-1519, DOI: 10.1080/09500690500186584

Levrini, O., Tasquier, G., Branchetti, L. y Barelli, E. (2019). Developing future-scaffolding skills through science education. International Journal of Science Education, 41(18), 2647-2674. DOI: 10.1080/09500693.2019.1693080

Mkimbili, S. T. y Ødegaard, M. (2019). Student Motivation in Science Subjects in Tanzania, Including Students' Voices. Research in Science Education, 49(6), 1835-1859. DOI: $10.1007 / \mathrm{s} 11165-017-9677-4$

Núñez, F., Banet, E., y Cordón, R. (2009). Capacidades del alumnado de Educación Secundaria Obligatoria para la elaboración e interpretación de gráficas. Enseñanza de las Ciencias: Revista de Investigación y Experiencias Didácticas, 27(3), 447-462.

OCDE (2006). PISA 2006: Marco de la evaluación. Conocimientos y habilidades en Ciencias, Matemáticas y Lectura.

Orden ECD/65/2015, de 21 de enero, por la que se describen las relaciones entre las competencias, los contenidos y los criterios de evaluación de la educación primaria, la educación secundaria obligatoria y el bachillerato. BOE, 25, 6986-7003.

Ratcliffe, M. (1999). Evaluation of abilities in interpreting media reports of scientific research. International Journal of Science Education, 21(10), 1085-1099. DOI: $10.1080 / 095006999290200$

Vázquez, Á. y Manassero, M. A. (2012). La selección de contenidos para enseñar naturaleza de la ciencia y tecnología (parte 2): Una revisión aplicada a los currículos de ciencias españoles. Revista Eureka sobre Enseñanza y Divulgación de las Ciencias. 9(1), 32-53. DOI: 10.25267/rev_eureka_ensen_divulg_cienc.2012.v9.i1.03

Zúñiga, A., Leiton, R. y Naranjo, J. A. (2014). Del sistema educativo tradicional hacia la formación por competencias: Una mirada a los procesos de enseñanza-aprendizaje de las ciencias en la educación secundaria de Mendoza, Argentina, y San José de Costa Rica. Revista Eureka sobre Enseñanza y Divulgación de las Ciencias, 11(2), 145-159. DOI: 10.25267/Rev_Eureka_ensen_divulg_cienc.2014.v11.i2.03

CÓMO CITAR ESTE ARTÍCULO

Franco López, A., Ayuso Fernández, G. E. y López-Banet, L. (2021). Evaluación de la adquisición de la competencia científica entre el alumnado de Biología de la ESO y una propuesta para mejorar su habilidad en las representaciones gráficas. Didáctica de las ciencias experimentales y sociales, 41, 89-118. DOI: 10.7203/DCES.41.19095 


\section{ANEXO 1. CUESTIONARIO SOBRE COMPETENCIA CIENTÍFICA BLOQUE I. ¿QUÉ CONOCEMOS SOBRE ALGUNOS CONCEPTOS IMPORTANTES EN LOS PROCESOS DE INVESTIGACIÓN?}

Seguramente habrás oído o leído algunos términos que desempeñan un papel muy importante en las investigaciones de los científicos; algunos de ellos, no todos, los habrás escuchado en clase o los habrás utilizado cuando has hablado con amigos o con tu familia.

1. ¿Recuerdas haber desarrollado actividades en clase -en este curso o en cursos anteriores- en las que tuvieras que identificar problemas o formular preguntas en una investigación? Elige una de las frases marcando a la izquierda con una $X$.
a. Con mucha frecuencia
b. Con alguna frecuencia
c. Casi nunca
d. No recuerdo haberlo hecho ninguna vez
e. No entiendo lo que se me pregunta
f. Otra:

2. ¿Recuerdas haber desarrollado actividades en clase -en este curso o en cursos anterioresen las que tuvieras que expresar hipótesis? Elige una de las frases marcando con una X.
a. Con mucha frecuencia
b. Con alguna frecuencia
c. Casi nunca
d. No recuerdo haberlo hecho ninguna vez
e. No entiendo lo que se me pregunta
f. Otra:

3. Variable es otro término habitual en las investigaciones que desarrollan los científicos, pero que también se utiliza en las clases de ciencias y de matemáticas. Nos gustaría saber si crees conocer el significado del término "variable":
a. Sí, sé lo que significa
b. Creo que sé lo que significa
c. Lo he oído algunas veces, pero no recuerdo su significado
d. No sé lo que significa variable
e. No entiendo lo que se me pregunta

4. El diseño experimental es un aspecto fundamental en una investigación científica. Nos gustaría saber si crees conocer el significado de la expresión "diseño experimental":
a. Sí, sé lo que significa
b. Creo que sé lo que significa
c. Lo he oído algunas veces, pero no recuerdo su significado
d. No sé lo que significa diseño experimental
e. No entiendo lo que se me pregunta

5. Marca con una X la frase (o frases) correctas: "Estamos haciendo un diseño experimental cuando...":
a. ... seguimos el guion que nos ha dado el profesor para observar células con el microscopio en el laboratorio de Ciencias.
b. ... proponemos las actividades a desarrollar para comprobar si es cierta la hipótesis de que de padres altos nacen hijos altos.
c. ... interpretamos los resultados de una experiencia para comprobar el efecto de la luz sobre el crecimiento de una planta. d. ... clasificamos plantas, con ayuda de unas claves del libro, por la forma y tamaño de sus hojas. 
BLOQUE II. ¿CÓMO RESOLVEMOS LAS SIGUIENTES CUESTIONES SOBRE INVESTIGACIONES?

1. Un estudio realizado por la Universidad de Murcia ha contado con una muestra de 772 personas entre 55 y 80 años de la Región repartidos en dos grupos. Al primero se le ha suministrado una dieta mediterránea enriquecida con aceite de oliva y al segundo se le ha proporcionado una dieta rica en grasas animales. A los 3 meses, en los dos casos, se ha medido la concentración de colesterol en sangre, un indicador de riesgo cardiovascular.

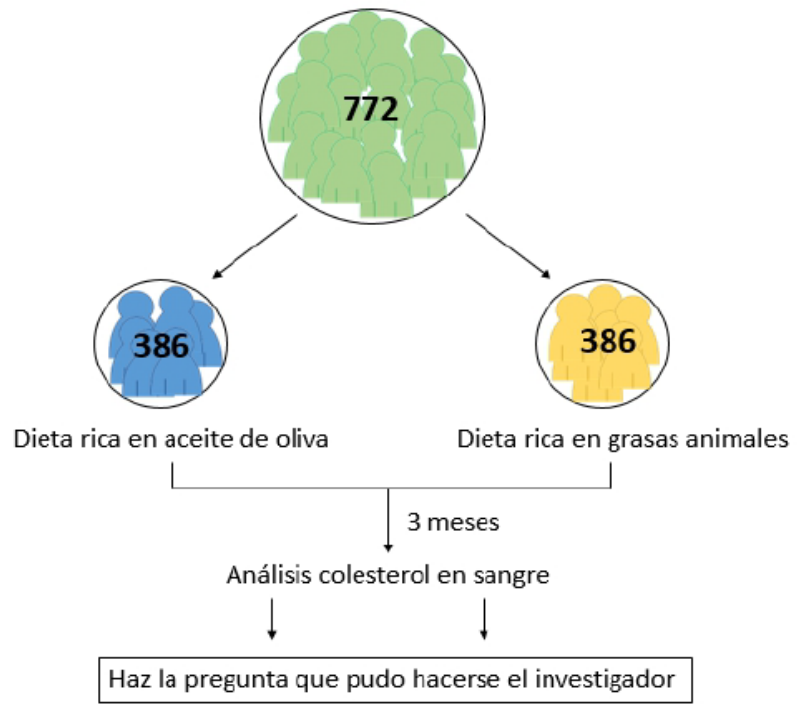

¿Qué se pretende investigar en este estudio? Haz la pregunta que pudo hacerse el investigador.

2. Las cochinillas son pequeños animales que viven escondidos, por ejemplo, debajo de la hojarasca. Decides averiguar si influye en ello los siguientes factores:

- luz / oscuridad

- temperatura alta (por ejemplo, 30 ㄷ) / temperatura baja (por ejemplo 10 ㄷ).

Imagina que tienes el material que necesites (cochinillas, cajas, hojarasca, dispositivos para regular la temperatura, etc.).

Escribe con todo el detalle que puedas, el experimento o los experimentos qué harías para demostrar si esos factores influyen en que las cochinillas estén escondidas.

a. Escribe el problema científico que se plantea en esta investigación:

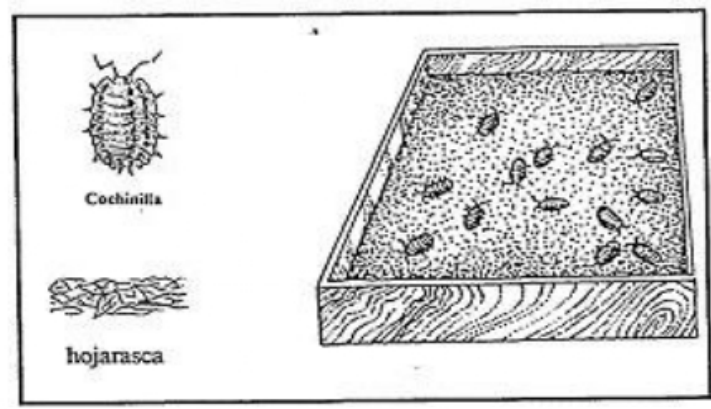

b. Escribe tu hipótesis o tus hipótesis para este problema:

c. Describe con todo el detalle que puedas, el experimento o los experimentos qué harías. Ayúdate con dibujos.

d. Indica la conclusión a la que crees que llegarías y justifica en qué te basarías. 
3. Hasta el siglo XVIII se pensaba que algunos seres vivos de pequeño tamaño (por ejemplo, insectos) se formaban en la carne putrefacta sin necesidad de que las hembras pusieran huevos. El italiano Francesco Redi en el año 1660 pensaba que eso no era posible e hizo el siguiente experimento para demostrarlo:

Cogió frascos que contenían trozos de carne, luego cerró la mitad de ellos y dejó abiertos la otra mitad. Las moscas sólo podían entrar en estos últimos y sólo en ellos se desarrollaron las larvas (pequeños gusanos que se transforman en moscas). En los frascos cerrados la carne se descompuso y se pudrió, pero no aparecieron larvas de mosca. Redi repitió la experiencia cubriendo los frascos con gasa, en lugar de cerrarlos herméticamente; de esta forma entraba aire a la carne, pero no las moscas. En este caso tampoco aparecieron larvas.

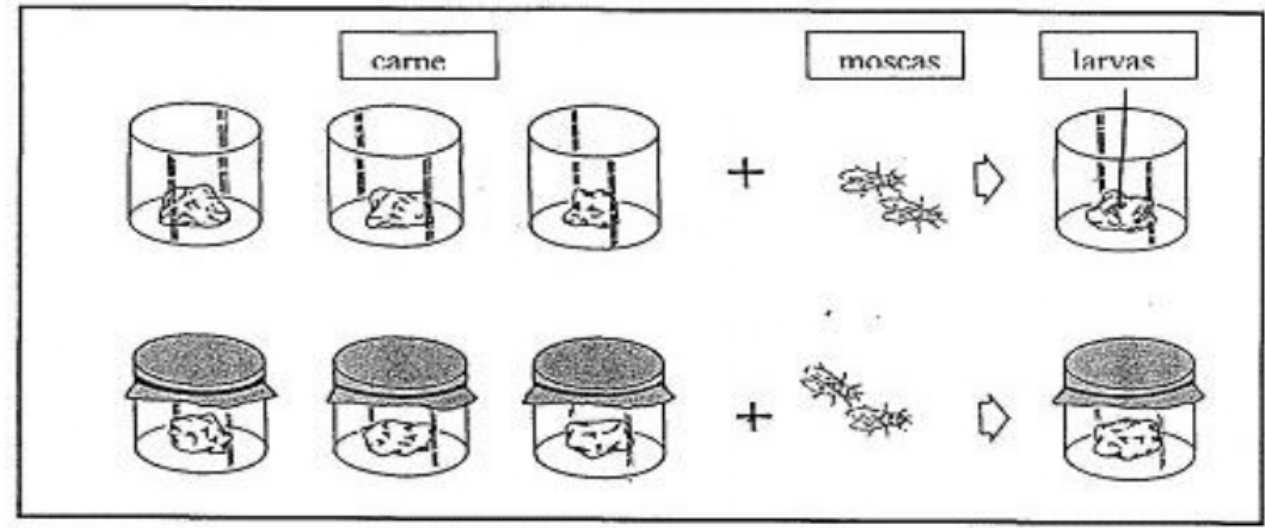

a. ¿Qué problema pretendía resolver Redi?

b. Escribe la hipótesis de este experimento.

c. ¿A qué conclusión llegó Redi?

4. Determinados peces de acuario de agua dulce sufren a menudo la denominada enfermedad del punto blanco, caracterizada por la presencia de pequeñas manchas blancas en las escamas y las aletas. Un grupo de investigación del Acuario de Murcia, que está analizando esta enfermedad, realiza un análisis de las manchas, observando la presencia en las mismas de un protozoo, un organismo unicelular microscópico.

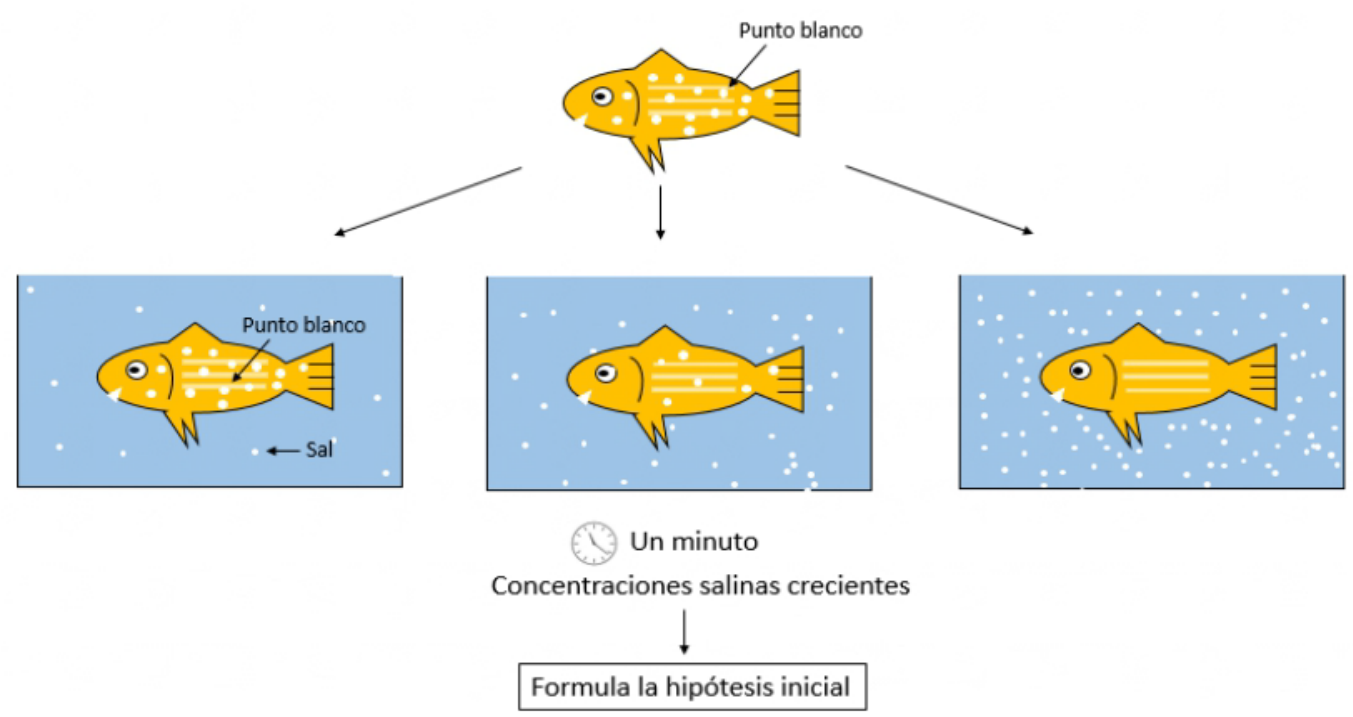


Este mismo grupo decide introducir durante unos minutos a los peces infectados en agua con una concentración salina superior a la del acuario, y observan que los protozoos desaparecen en la mayor parte de los casos.

La tabla siguiente muestra los resultados del experimento realizado con peces de agua dulce del acuario que padecían la enfermedad del punto blanco cuando eran sumergidos durante un minuto en soluciones salinas a diversas concentraciones.

\begin{tabular}{|l|c|c|c|c|c|c|c|c|c|c|}
\hline Concentración salina (g/L) & 2.5 & 5.0 & 7.5 & 10 & 12.5 & 15.0 & 17.5 & 20.0 & 22.5 & 25.0 \\
\hline Número de protozoos & 150 & 150 & 150 & 146 & 140 & 100 & 45 & 10 & 8 & 0 \\
\hline
\end{tabular}

Nota: $\mathrm{g} / \mathrm{L}$ es la unidad en la que se mide la concentración de sal.

Formula la hipótesis inicial del problema del acuario:

5. Durante un trabajo escolar unos estudiantes hicieron un anillo de agua salada alrededor de un caracol y observaron que no lo atravesaba. Según algunos se debía al agua, según otros a la sal.

¿Qué pruebas harías para averiguar quién tiene razón? Explícalo con detalle.

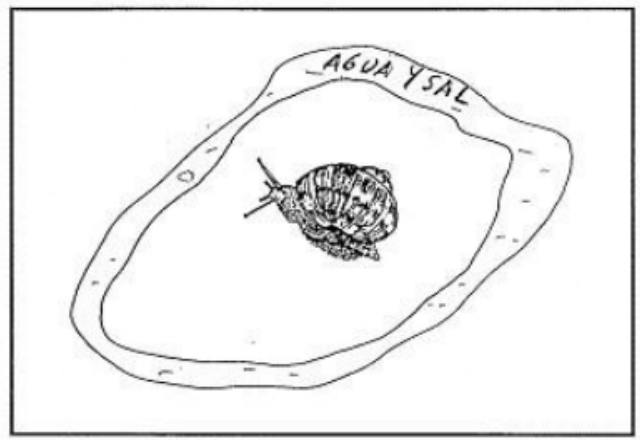

6. En el curso 2018-19 se propuso, a alumnos de 3 ESO, una investigación sobre la germinación (inicio del crecimiento de una planta a partir de una semilla) de unas semillas que eran desconocidas para ellos. Uno de los grupos decidió averiguar cuál era la temperatura mejor para su germinación. En su cuaderno escribieron lo siguiente:

"Pensábamos que lo normal sería que germinasen como otras semillas, a una temperatura normal, ni muy fría ni muy caliente. Pusimos en el laboratorio una maceta grande, con tierra buena, y sembramos 30 semillas. Durante los 7 días que duró el experimento, dejamos las persianas subidas para que hubiera luz suficiente y se regó la maceta todos los días un poco. La temperatura media en el laboratorio fue, durante esos días de 22ㅇ. A los 3 días empezaron a brotar algunas semillas. A los 7 días germinaron ya 25 de las 30 semillas.

La conclusión que sacamos fue que la temperatura media mejor para germinar esas semillas es de unos $22 \circ \mathrm{C}^{\prime \prime}$

El experimento de estos alumnos no permite llegar a la conclusión que hicieron. ¿En qué se equivocaron? 
7. La profesora de Biología y Geología te pide realizar un trabajo de investigación para profundizar en la relación que existe entre la edad y la talla de las personas de tu familia. Al rellenar la tabla obtienes los datos que aparecen en el margen derecho.

Realiza una gráfica donde expongas los datos recogidos.

\begin{tabular}{|c|c|}
\hline Años (edad) & Talla (cm) \\
\hline 10 & 125 \\
\hline 15 & 150 \\
\hline 20 & 175 \\
\hline 25 & 178 \\
\hline 70 & 175 \\
\hline
\end{tabular}

8. En el gráfico siguiente se muestra un estudio realizado en el año 2017 por la Consejería de Sanidad de la Región de Murcia sobre jóvenes estudiantes y su consumo de alcohol semanal. Basándote en la gráfica, contesta a las preguntas que se te plantean:

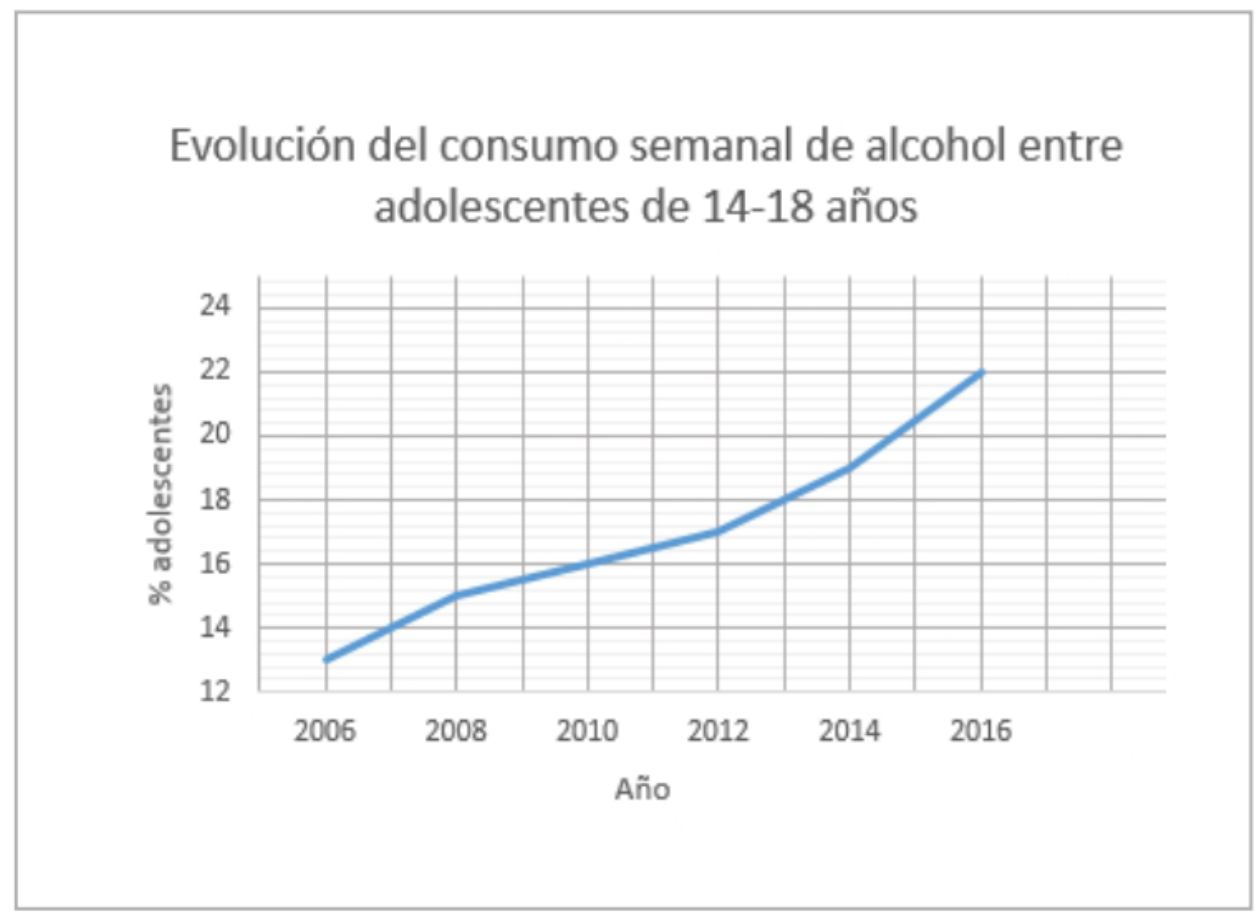

a. ¿Cuál es el porcentaje de adolescentes que consumían alcohol semanalmente en el año 2013?

b. ¿Cuál es el aumento de porcentaje en el consumo de alcohol semanal entre los años 2010 y 2016?

c. Si la tendencia fue la misma, ¿cuál pudo ser el porcentaje de jóvenes que consumió alcohol en el 2018?

d. ¿Cómo ha variado el porcentaje de jóvenes que consumen alcohol semanalmente con el paso de los años? 


\section{ANEXO 2. ACTIVIDAD 1. BIENVENIDOS AL GRUPO}

Tras recibir tu respuesta a la carta anterior, Armando, el epidemiólogo del centro de Coordinación de Alertas y Emergencias Sanitarias, te manda el siguiente correo para que podáis empezar a trabajar:

ArmandoZanón < institutoepidemiologico@gmail.com >

Buenos días,

Me alegra ver que habéis aceptado nuestra propuesta. Siento no poder acudir personalmente a entregaros los resultados, pero entenderéis que, dada la situación actual y el gran volumen de trabajo, me es imposible.

Para empezar, me gustaría hacer un estudio donde se refleje el número de personas afectadas por edades. ¿Cuál creéis que sería el etiquetado correcto? Señálalo con un círculo.

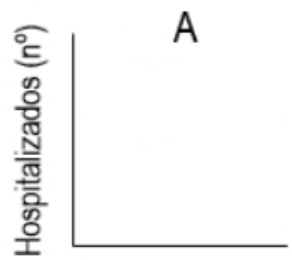

Día

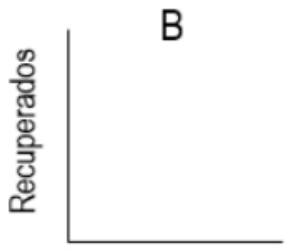

Edad

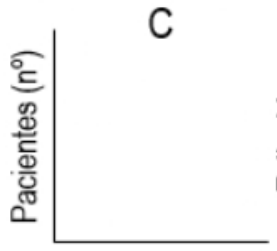

Sexo

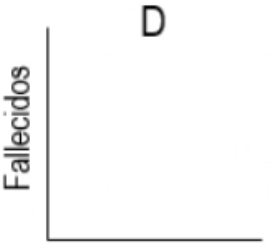

Edad

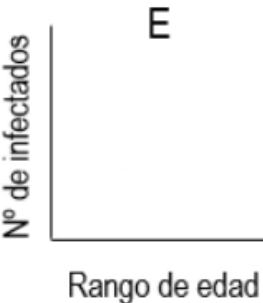

Figura 1. Buscando el etiquetado correcto.

A los pocos minutos, Armando vuelve a escribirte un correo electrónico con una serie de preguntas nuevas:

Armando Zanón < institutoepidemiologico@gmail.com > 10:09

Tras leer vuestro mensaje me han surgido nuevas preguntas a las que me gustaría que contestarais:

¿En qué os habéis basado para elegir esa opción y descartar las otras?

¿Qué información obtendrías de cada una de las gráficas anteriores según los ejes?

En caso de haber cambiado la elección inicial, comunicármelo sin problema. 
Armando Zanón < institutoepidemiologico@gmail.com > $10: 41$

¡Hola de nuevo! Os escribo para mandaros una información de última hora.

Estamos analizando el número de nuevos casos en Murcia durante los primeros días. Sin embargo, los diferentes grupos de investigación parecen no ponerse de acuerdo con la representación gráfica. ¿Qué dos gráficas representan la misma información? ¿En qué te has fijado? ¿Qué indica cada gráfica? (Apóyate en los datos).

\section{Nuevos casos por día}

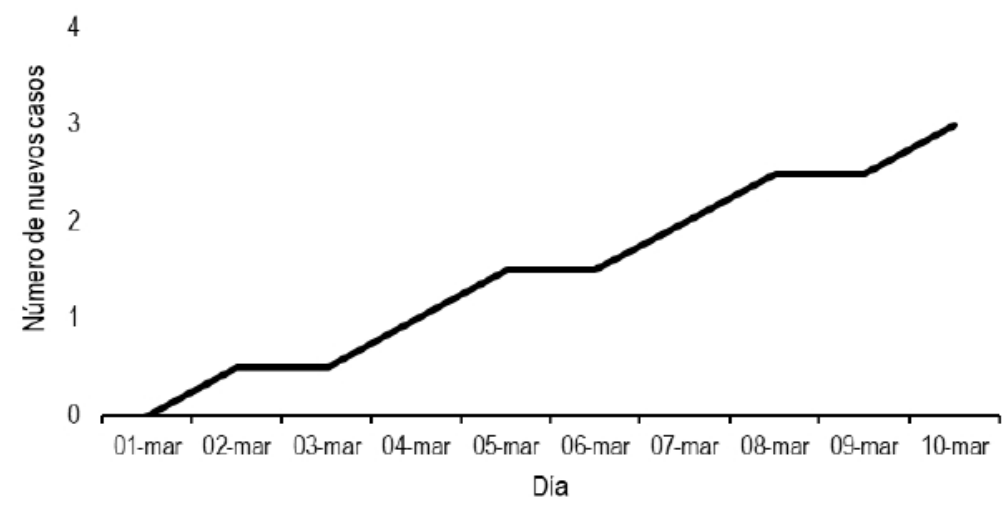

Nuevos casos por día

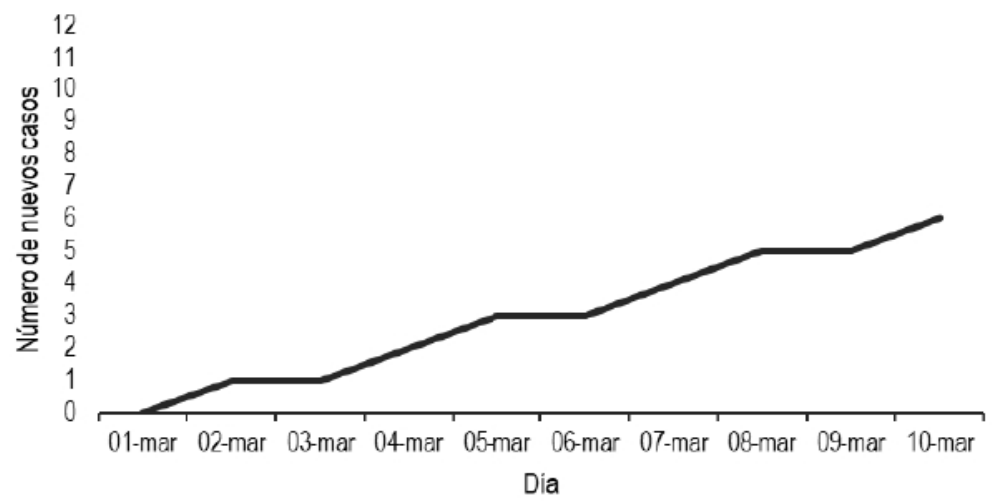

Nuevos casos por día

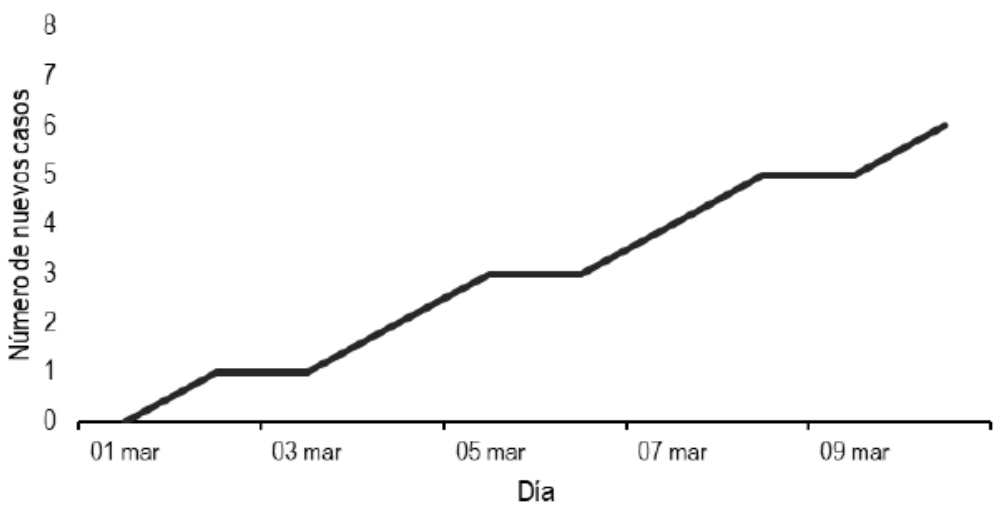

Figura 2. Nuevos casos por día en la Región. 


\section{ANEXO 3. ACTIVIDAD 2. LOS CASOS SE DISPARAN EN LA REGIÓN}

Armando os vuelve a escribir para proporcionaros nuevos datos de la epidemia que está azotando a la Región:

¡Buenos días compañeros!

Pensábamos que se trataba de un brote restringido a la capital, sin embargo, estamos observando casos con sintomatología parecida en ciertas comarcas de la Región. En otras, como la Vega del Segura, el Bajo Guadalentín y las Cuencas de Abanilla, Fortuna y Mula, el número de casos registrados hasta el momento es cero. Me gustaría construyerais una gráfica donde aparezca la información del siguiente mapa:

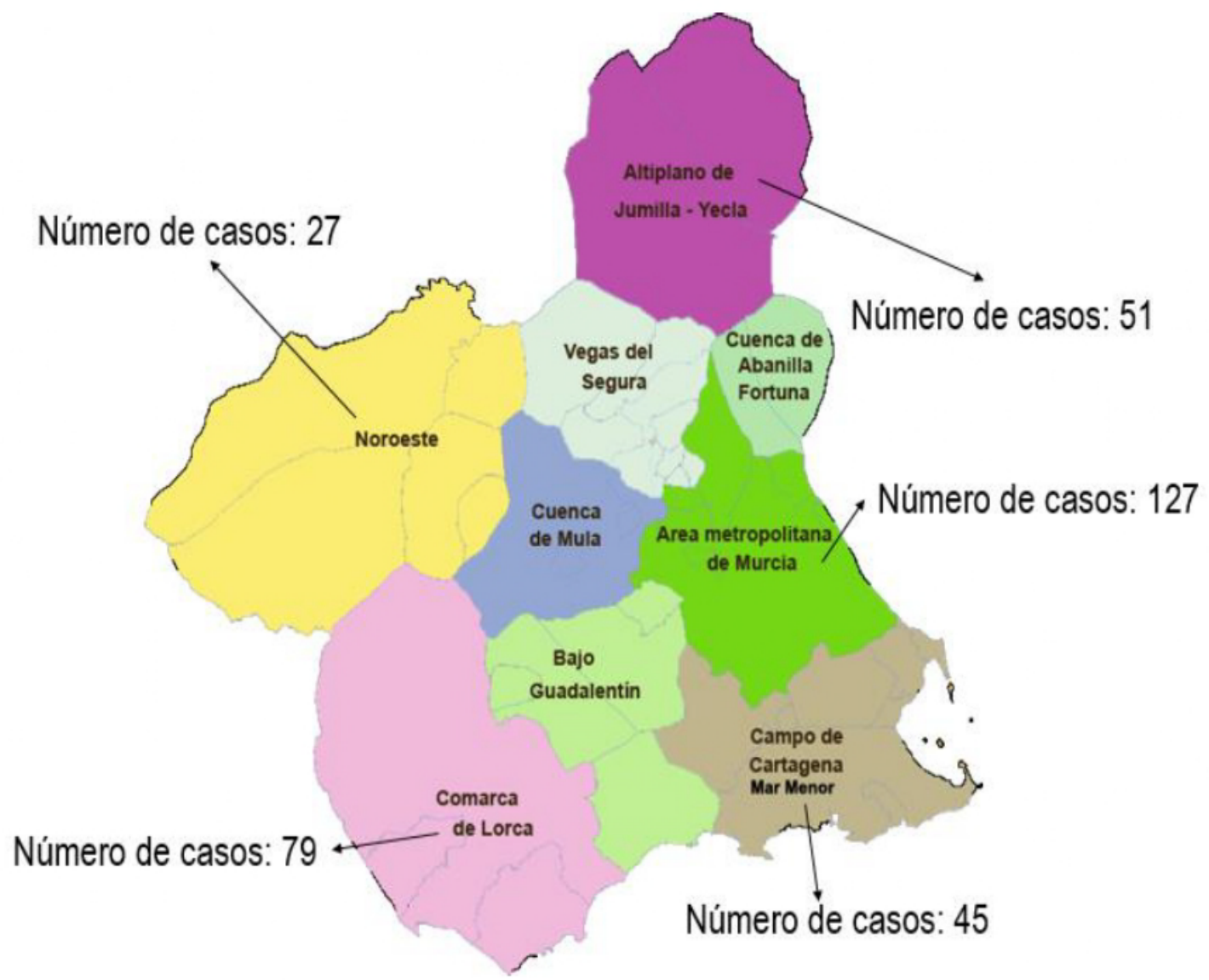

Antes de realizar la gráfica responder a estas cuestiones:

\begin{tabular}{l|l}
\hline ¿Qué elementos & \\
componen las gráficas? & \\
\hline ¿Cómo construiríais una & \\
tabla para exponer los & \\
datos que aparecen en el & \\
mapa? & \\
\hline ¿Cuál es la finalidad de la & \\
gráfica? ¿Y de realizar una & \\
tabla antes? & \\
\hline
\end{tabular}


Antes de realizar la gráfica en el cuadro que aparece más abajo, creo que puede seros muy útil responder a las siguientes preguntas:

\section{1) Elección del gráfico}

¿Qué tipo de gráfico usarías para representar los datos del mapa ( $A, B, C$ u otro) ?
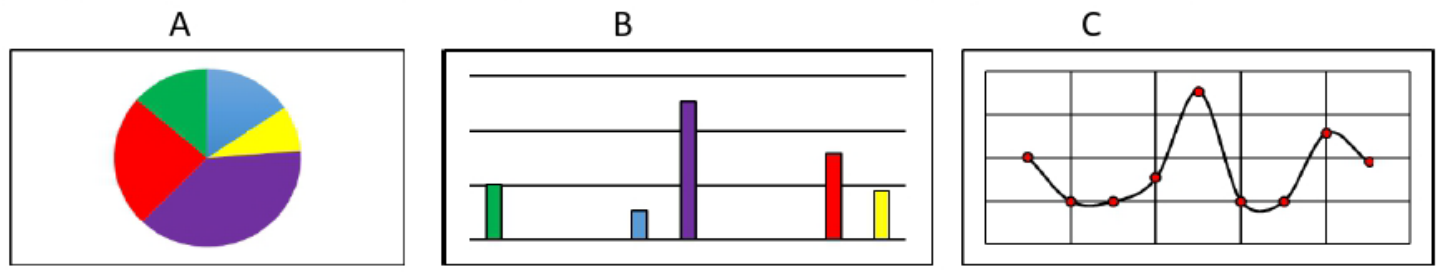

¿Qué motivos te han hecho elegir ese y descartar los otros dos? ¿Cómo crees que podrían mejorarse los gráficos anteriores?

\section{2) Proporción}

¿Cuál es el dato mayor? ¿Y el menor? ¿Debéis de tenerlo en cuenta? ¿Por qué lo creéis? ¿Qué serie van a seguir los ejes de la gráfica (de 5 en 5, de 10 en 10, de 25 en 25...)? Pensad que siempre deben seguir ese patrón.

En caso de ser un gráfico circular (A), ¿qué deberías tener en consideración?

\section{3) Ejes y títulos de la gráfica}

¿Qué título va a tener vuestra gráfica? ¿Por qué habéis elegido ese?

Si los hubiera, ¿qué título van a tener los ejes? ¿Hay unidades? En caso afirmativo, ¿deberías ponerlas? ¿Dónde debéis escribirlas?

¿Puede que una leyenda ayude a entender vuestra gráfica a la gente del Centro de Alertas y Emergencias Sanitarias? Explica razonadamente tu respuesta. 
¡Hola de nuevo!

Una vez estudiadas las zonas geográficas, queremos ver porque algunas zonas tienen un mayor número de casos que otras. Para ello, estamos siguiendo la pista a 4 lugares al que acudieron muchas personas, entre ellos varios contagiados, el miércoles, 10 días antes de que todo esto empezara:

\begin{tabular}{l|l|c|c}
\hline \multicolumn{1}{c|}{ Lugar } & \multicolumn{1}{c|}{ Zona afectada } & $\begin{array}{c}\text { No de personas } \\
\text { contagiadas }\end{array}$ & $\begin{array}{c}\text { No de personas } \\
\text { que acudieron }\end{array}$ \\
\hline Cine & $\begin{array}{l}\text { Área metropolitana, campo de Cartagena, } \\
\text { cuenca de Mula y Comarca de Lorca }\end{array}$ & 0 & 565 \\
\hline $\begin{array}{l}\text { Fiestas } \\
\text { privadas }\end{array}$ & $\begin{array}{l}\text { Área metropolitana, Comarca de Lorca, } \\
\text { noroeste y altiplano de la Región y Campo } \\
\text { de Cartagena. }\end{array}$ & 36 & 188 \\
\hline Institutos & $\begin{array}{l}\text { Noroeste de la Región, altiplano de } \\
\text { Jumilla-Yecla, Bajo Guadalentín, Vegas del } \\
\text { Segura, Comarca de Lorca }\end{array}$ & 66 & 37.598 \\
\hline Jardines & Área metropolitana. & 7 & \\
\hline
\end{tabular}

Buscad un punto en común entre los datos de la tabla y la gráfica que habéis construido.

Parece que muchas personas se contagiaron en esos lugares, pero ¿cómo pudieron contagiarse el resto de individuos? ¿Qué lugar es el foco más peligroso? ¿En qué os habéis basado para dar ese dato?

Escribe una carta al vecindario de las zonas afectadas, explicándoles la gravedad de la situación, zonas más afectadas, consecuencias y otros datos que consideréis de interés. Para redactarla, apoyaros en los datos.

A la atención de los vecinos de

Nos dirigimos a ustedes para... 


\section{ANEXO 4. ACTIVIDAD 3: “DANDO CON EL CULPABLE” (FLASHCARD)}

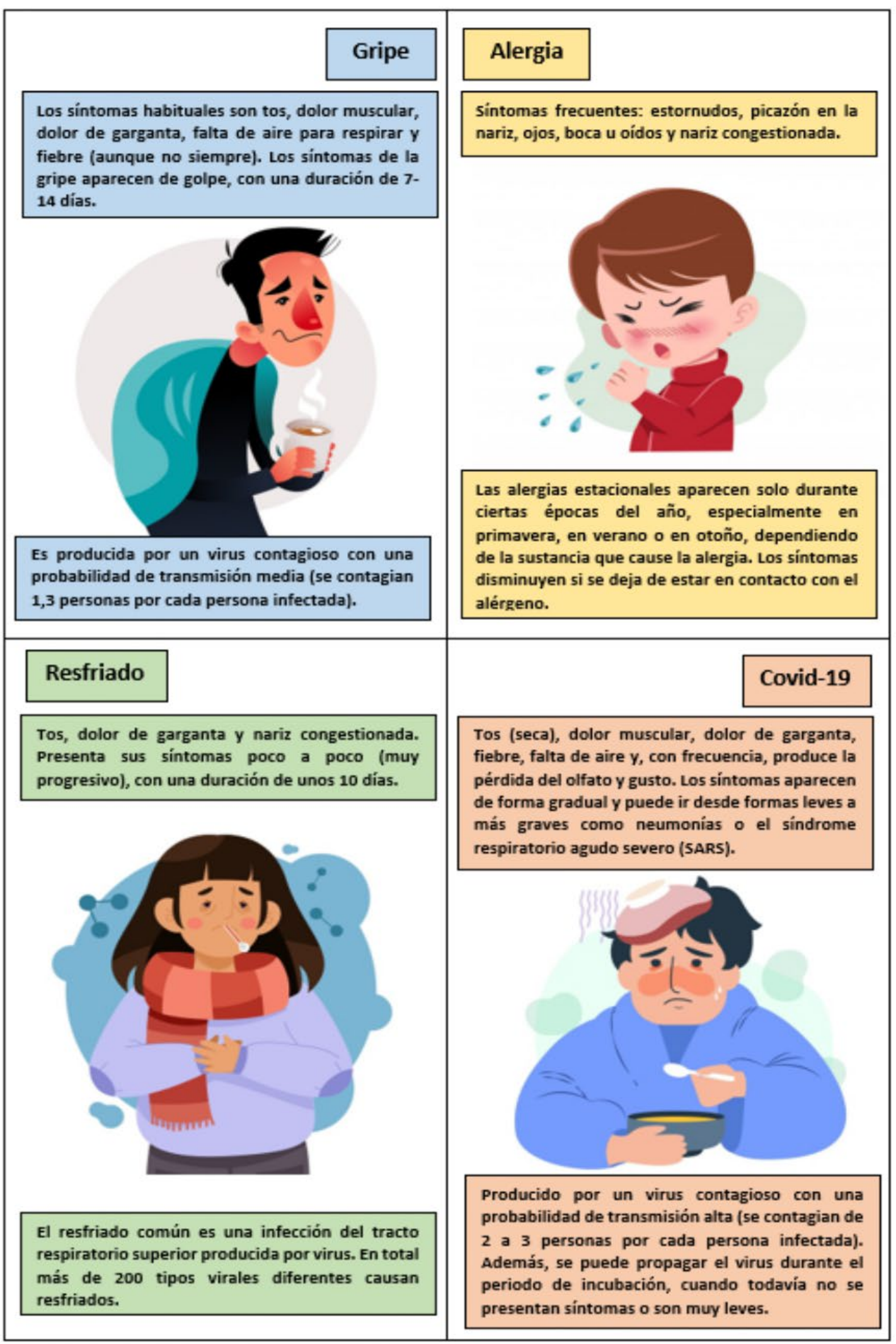




\section{ANEXO 5. ACTIVIDAD 4. LA EDAD Y EL SEXO, ¿UN FACTOR ENTRE LOS CONTAGIADOS?}

Armando vuelve a contactar con vosotros por carta para mandaros las últimas tareas de la investigación que venís desarrollando.

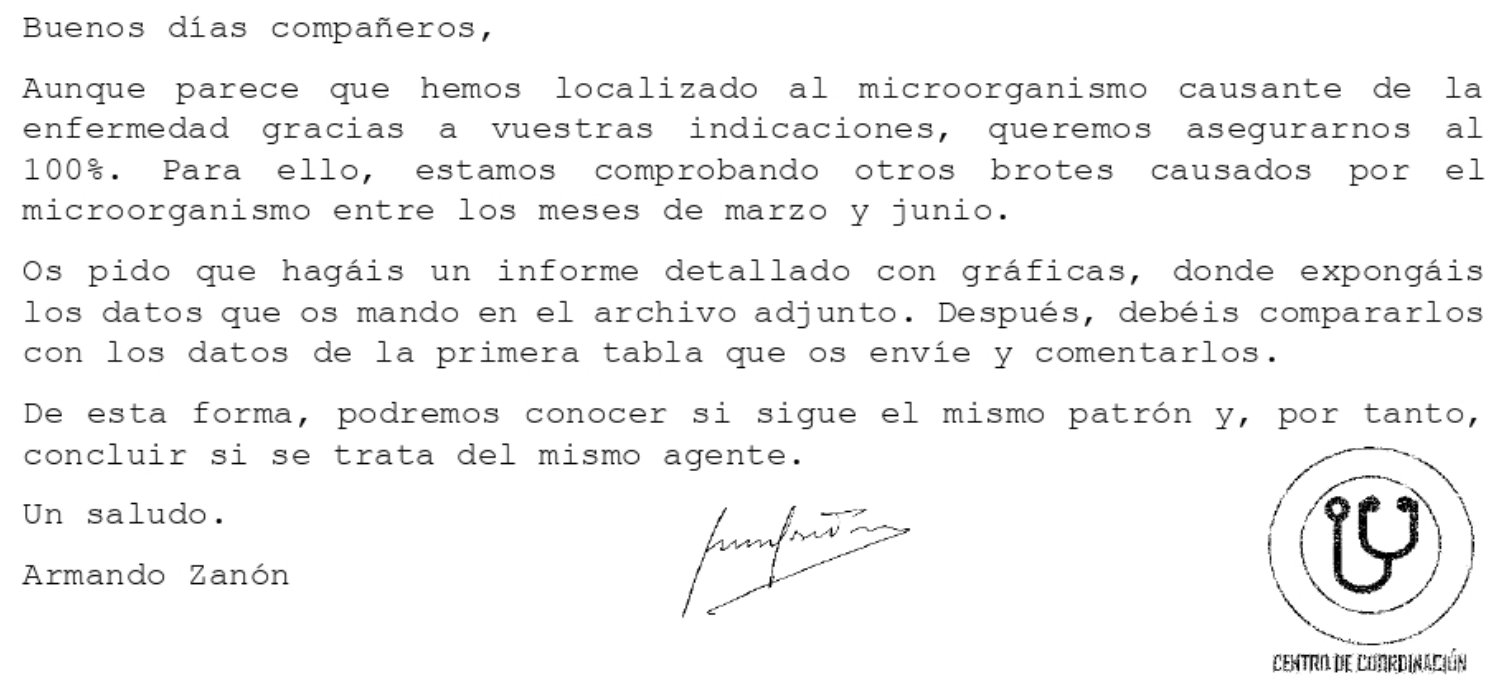

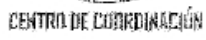

Archivo adjunto:

1. Personas afectadas por sexos entre los meses de marzo y junio:

2. Personas afectadas por edades entre los meses de marzo y junio

\begin{tabular}{|c|c|}
\hline Sexo & Porcentaje \\
\hline Hombre (H) & $59.0 \%$ \\
\hline Mujer (M) & $41.0 \%$ \\
\hline
\end{tabular}

\begin{tabular}{|c|c|}
\hline Rango de edad & Porcentaje \\
\hline$\leq 15$ & $6.5 \%$ \\
\hline $15-44$ & $16.6 \%$ \\
\hline $44-65$ & $26.9 \%$ \\
\hline$\geq 65$ & $50.0 \%$ \\
\hline
\end{tabular}

A continuación, debéis realizar en grupos un informe final en el que comentéis cada una de las gráficas y responder a las preguntas que aparecen más abajo.

\begin{tabular}{|l|l|}
\hline \multicolumn{1}{|c|}{ GRÁFICA 1 } & \multicolumn{1}{|c|}{ GRÁFICA 2 } \\
\hline & \\
& \\
Comentarios: & Comentarios: \\
\hline
\end{tabular}

¿A qué conclusión llegáis sobre el problema planteado?

¿Qué habéis aprendido a lo largo de la resolución del problema? 
